\title{
Generalized sampling: From shift-invariant to $U$-invariant spaces
}

\author{
H. R. Fernández-Morales and A. G. García
}

M. A. Hernández-Medina

M. J. Muñoz-Bouzo

The aim of this article is to derive a sampling theory in $U$-invariant subspaces of a separable Hilbert space $\mathcal{H}$ where $U$ denotes a unitary operator defined on $\mathcal{H}$. To this end, we use some special dual frames for $L^{2}(0,1)$, and the fact that any $U$-invariant subspace with stable generator is the image of $L^{2}(0,1)$ by means of a bounded invertible operator. The used mathematical technique mimics some previous sampling work for shift-invariant subspaces of $L^{2}(\mathbb{R})$. Thus, sampling frame expansions in $U$-invariant spaces are obtained. In order to generalize convolution systems and deal with the timejitter error in this new setting we consider a continuous group of unitary operators which includes the operator $U$.

Keywords: Stationary sequences; $U$-invariant subspaces; frames; dual frames; time-jitter error; group of unitary operators; pseudo-dual frames.

\section{By Way of Motivation}

The aim in this paper is to derive a generalized sampling theory for $U$-invariant subspaces of a separable Hilbert space $\mathcal{H}$, where $U: \mathcal{H} \rightarrow \mathcal{H}$ denotes a unitary 
operator. The motivation for our work can be found in the generalized sampling problem in shift-invariant subspaces of $L^{2}(\mathbb{R})$; there $\mathcal{H}:=L^{2}(\mathbb{R})$ and the unitary operator is the shift $T: f(u) \mapsto f(u-1)$ in $L^{2}(\mathbb{R})$. In that setting, the functions (signals) belong to some (principal) shift-invariant subspace $V_{\varphi}^{2}:=\overline{\operatorname{span}}_{L^{2}(\mathbb{R})}\{\varphi(u-n)$, $n \in \mathbb{Z}\}$, where the generator function $\varphi$ belongs to $L^{2}(\mathbb{R})$ and the sequence $\{\varphi(u-n)\}_{n \in \mathbb{Z}}$ is a Riesz sequence for $L^{2}(\mathbb{R})$. Thus, the shift-invariant space $V_{\varphi}^{2}$ can be described as

$$
V_{\varphi}^{2}=\left\{\sum_{n \in \mathbb{Z}} \alpha_{n} \varphi(u-n):\left\{\alpha_{n}\right\}_{n \in \mathbb{Z}} \in \ell^{2}(\mathbb{Z})\right\} .
$$

On the other hand, in many common situations the available data are samples of some filtered versions $f * h_{j}$ of the signal $f$ itself, where the average function $h_{j}$ reflects the characteristics of the adquisition device.

For $s$ convolution systems (linear time-invariant systems or filters in engineering jargon) $\mathcal{L}_{j} f:=f * \mathrm{~h}_{j}, j=1,2, \ldots, s$, defined on $V_{\varphi}^{2}$, and assuming also that the sequence of samples

$$
\left\{\left(\mathcal{L}_{j} f\right)(r m)\right\}_{m \in \mathbb{Z} ; j=1,2, \ldots, s},
$$

where $r \in \mathbb{N}$, is available for any $f$ in $V_{\varphi}^{2}$, the generalized sampling problem mathematically consists of the stable recovery of any $f \in V_{\varphi}^{2}$ from the above sequence of samples. In other words, it deals with the construction of sampling formulas in $V_{\varphi}^{2}$ having the form

$$
f(u)=\sum_{j=1}^{s} \sum_{m \in \mathbb{Z}}\left(\mathcal{L}_{j} f\right)(r m) S_{j}(u-r m), \quad u \in \mathbb{R},
$$

where the sequence of reconstruction functions $\left\{S_{j}(\cdot-r m)\right\}_{m \in \mathbb{Z} ; j=1,2, \ldots, s}$ is a frame for the shift-invariant space $V_{\varphi}^{2}$.

Sampling in shift-invariant spaces of $L^{2}(\mathbb{R})$ (or $L^{2}\left(\mathbb{R}^{d}\right)$ ), with one or multiple generators, has been profusely treated in the mathematical literature. A few selected references are: $[4,5,9-14,18,23,27-31]$.

In this work we provide a generalization of the above problem in the following sense. Let $U$ be a unitary operator in a separable Hilbert space $\mathcal{H}$; for a fixed $a \in \mathcal{H}$, consider the closed subspace given by $\mathcal{A}_{a}:=\overline{\operatorname{span}}\left\{U^{n} a, n \in \mathbb{Z}\right\}$. In case that the sequence $\left\{U^{n} a\right\}_{n \in \mathbb{Z}}$ is a Riesz sequence in $\mathcal{H}$ we have

$$
\mathcal{A}_{a}=\left\{\sum_{n \in \mathbb{Z}} \alpha_{n} U^{n} a:\left\{\alpha_{n}\right\}_{n \in \mathbb{Z}} \in \ell^{2}(\mathbb{Z})\right\} .
$$

In order to generalize convolution systems and mainly to obtain some perturbation results in this new setting, we assume that the operator $U$ is included in a continuous group of unitary operators $\left\{U^{t}\right\}_{t \in \mathbb{R}}$ in $\mathcal{H}$ as $U:=U^{1}$. Recall that $\left\{U^{t}\right\}_{t \in \mathbb{R}}$ is a family of unitary operators in $\mathcal{H}$ satisfying (see [2, Vol. 2; p. 29]):

(1) $U^{t} U^{t^{\prime}}=U^{t+t^{\prime}}$ 
(2) $U^{0}=I_{\mathcal{H}}$,

(3) $\left\langle U^{t} x, y\right\rangle_{\mathcal{H}}$ is a continuous function of $t$ for any $x, y \in \mathcal{H}$.

Note that $\left(U^{t}\right)^{-1}=U^{-t}$, and since $\left(U^{t}\right)^{*}=\left(U^{t}\right)^{-1}$, we have $\left(U^{t}\right)^{*}=U^{-t}$.

Thus, for $b \in \mathcal{H}$ we consider the linear operator $\mathcal{H} \ni x \mapsto \mathcal{L}_{b} x \in C(\mathbb{R})$ such that $\left(\mathcal{L}_{b} x\right)(t):=\left\langle x, U^{t} b\right\rangle_{\mathcal{H}}$ for every $t \in \mathbb{R}$. These operators $\mathcal{L}_{b}$, which will be called $U$-systems, can be seen as a generalization of the convolution systems in $L^{2}(\mathbb{R})$. Indeed, for the shift operator $T: f(u) \mapsto f(u-1)$ in $L^{2}(\mathbb{R})$ we have

$$
\left\langle f, T^{t} b\right\rangle_{L^{2}(\mathbb{R})}=\int_{-\infty}^{\infty} f(u) \overline{b(u-t)} d u=(f * h)(t), \quad t \in \mathbb{R},
$$

where $h(u):=\overline{b(-u)}$.

Given $U$-systems $\mathcal{L}_{j}, j=1,2, \ldots, s$, corresponding to $s$ elements $b_{j} \in \mathcal{H}$, i.e. $\mathcal{L}_{j} \equiv \mathcal{L}_{b_{j}}$ for each $j=1,2, \ldots, s$, the generalized regular sampling problem in $\mathcal{A}_{a}$ consists of the stable recovery of any $x \in \mathcal{A}_{a}$ from the sequence of the samples

$$
\left\{\mathcal{L}_{j} x(r m)\right\}_{m \in \mathbb{Z} ; j=1,2, \ldots, s} \text { where } r \in \mathbb{N}, \quad r \geq 1 .
$$

This $U$-sampling problem has been treated, for the first time, in some recent papers $[22,24]$. Sampling in shift-invariant subspaces or in modulation-invariant subspaces of $L^{2}(\mathbb{R})$ becomes a particular case of $U$-sampling associated with the shift operator $T: f(u) \mapsto f(u-1)$ or with the modulation operator $M: f(u) \mapsto \mathrm{e}^{2 \pi \mathrm{i} u} f(u)$ in $L^{2}(\mathbb{R})$ respectively.

In this paper, we propose a completely different approach which allows to analyze in depth the $U$-sampling problem. In Sec. 3 , we prove the existence of frames in $\mathcal{A}_{a}$, having the form $\left\{U^{r m} c_{j}\right\}_{m \in \mathbb{Z} ; j=1,2, \ldots, s}$, where $c_{j} \in \mathcal{A}_{a}$ for $j=1,2, \ldots, s$, such that for each $x \in \mathcal{A}_{a}$ the sampling expansion

$$
x=\sum_{j=1}^{s} \sum_{m \in \mathbb{Z}} \mathcal{L}_{j} x(r m) U^{r m} c_{j} \text { in } \mathcal{H}
$$

holds. To this end, as in the shift-invariant case (see, for instance, $[13,14]$ ), we use that the above sampling formula is intimately related with some special dual frames in $L^{2}(0,1)$ (see Sec. 2) via the isomorphism $\mathcal{T}_{U, a}: L^{2}(0,1) \rightarrow \mathcal{A}_{a}$ which maps the orthonormal basis $\left\{\mathrm{e}^{2 \pi i n w}\right\}_{n \in \mathbb{Z}}$ for $L^{2}(0,1)$ onto the Riesz basis $\left\{U^{n} a\right\}_{n \in \mathbb{Z}}$ for $\mathcal{A}_{a}$. In [24], regular sampling expansions like (1.1) are obtained by using a completely different technique; basically, they use the cross-covariance function $R_{a, b_{j}}(n):=$ $\left\langle U^{n} a, b_{j}\right\rangle_{\mathcal{H}}$ between the sequences $\left\{U^{n} a\right\}_{n \in \mathbb{Z}}$ and $\left\{U^{n} b_{j}\right\}_{n \in \mathbb{Z}}, j=1,2, \ldots, s$.

Strictly speaking, we do not need the formalism of the continuous group of unitary operators to derive the sampling results in Sec. 3 since we only use the discrete group $\left\{U^{n}\right\}_{n \in \mathbb{Z}}$ completely determined by $U$. However, for the study, in Sec. 4, of the time-jitter error in sampling formulas as in (1.1), the continuous group of unitary operators $\left\{U^{t}\right\}_{t \in \mathbb{R}}$ becomes essential. In this case, we dispose of a perturbed sequence of samples $\left\{\left(\mathcal{L}_{j} x\right)\left(r m+\epsilon_{m j}\right)\right\}_{m \in \mathbb{Z} ; j=1,2, \ldots, s}$, with errors $\epsilon_{m j} \in \mathbb{R}$, for the recovery of $x \in \mathcal{A}_{a}$. We prove that, for small enough errors $\epsilon_{m j}$, 
the stable recovery of any $x \in \mathcal{A}_{a}$ is still possible. Finally, in Sec. 5 we deal with the case of multiple stable generators. We only sketch the procedure since it is essentially identical to the one-generator case.

\section{On Sampling in $U$-Invariant Subspaces}

For a fixed $a \in \mathcal{H}$, assume that the sequence $\left\{U^{n} a\right\}_{n \in \mathbb{Z}}$ is a Riesz sequence in $\mathcal{H}$. Recall that a Riesz basis in a separable Hilbert space is the image of an orthonormal basis by means of a bounded invertible operator. Any Riesz basis $\left\{x_{n}\right\}_{n \in \mathbb{Z}}$ has a unique biorthogonal (dual) Riesz basis $\left\{y_{n}\right\}_{n \in \mathbb{Z}}$, i.e. $\left\langle x_{n}, y_{m}\right\rangle_{\mathcal{H}}=\delta_{n, m}$, such that the expansions

$$
x=\sum_{n \in \mathbb{Z}}\left\langle x, y_{n}\right\rangle_{\mathcal{H}} x_{n}=\sum_{n \in \mathbb{Z}}\left\langle x, x_{n}\right\rangle_{\mathcal{H}} y_{n},
$$

hold for every $x \in \mathcal{H}$. We state the definition by considering the integers set $\mathbb{Z}$ as the index set since throughout the paper most of sequences are indexed in $\mathbb{Z}$. A Riesz sequence in $\mathcal{H}$ is a Riesz basis for its closed span (see, for instance, [8]). Thus, the $U$-invariant subspace $\mathcal{A}_{a}:=\overline{\operatorname{span}}\left\{U^{n} a, n \in \mathbb{Z}\right\}$ can be expressed as

$$
\mathcal{A}_{a}=\left\{\sum_{n \in \mathbb{Z}} \alpha_{n} U^{n} a:\left\{\alpha_{n}\right\}_{n \in \mathbb{Z}} \in \ell^{2}(\mathbb{Z})\right\} .
$$

For simplicity and ease of notation we are considering the one-generator setting; as we have already said, the same sampling results for the general case can be obtained by analogy, and it will be drawn in Sec. 5 . The sequence $\left\{U^{n} a\right\}_{n \in \mathbb{Z}}$ is a stationary sequence since the inner product $\left\langle U^{n} a, U^{m} a\right\rangle_{\mathcal{H}}$ depends only on the difference $n-m \in \mathbb{Z}$. Moreover, the auto-covariance $R_{a}$ of the sequence $\left\{U^{n} a\right\}_{n \in \mathbb{Z}}$ admits the integral representation

$$
R_{a}(k):=\left\langle U^{k} a, a\right\rangle_{\mathcal{H}}=\frac{1}{2 \pi} \int_{-\pi}^{\pi} \mathrm{e}^{\mathrm{i} k \theta} d \mu_{a}(\theta), \quad k \in \mathbb{Z},
$$

in terms of a positive Borel measure $\mu_{a}$ on $(-\pi, \pi)$ called the spectral measure of the sequence (see [19]). This is obtained from the integral representation of the unitary operator $U$ on $\mathcal{H}$ (see, for instance, $[2,33]$ ). The spectral measure $\mu_{a}$ can be decomposed into an absolute continuous and a singular part as $d \mu_{a}(\theta)=$ $\phi_{a}(\theta) d \theta+d \mu_{a}^{s}(\theta)$. A necessary and sufficient condition in order for the sequence $\left\{U^{n} a\right\}_{n \in \mathbb{Z}}$ to be a Riesz sequence for $\mathcal{H}$ is given in next theorem in terms of the decomposition of the spectral measure $\mu_{a}$.

Theorem 2.1. Let $\left\{U^{n} a\right\}_{n \in \mathbb{Z}}$ be a sequence obtained from a unitary operator in a separable Hilbert space $\mathcal{H}$ with spectral measure $d \mu_{a}(\theta)=\phi_{a}(\theta) d \theta+d \mu_{a}^{s}(\theta)$, and let $\mathcal{A}_{a}$ be the closed subspace spanned by $\left\{U^{n} a\right\}_{n \in \mathbb{Z}}$. Then the sequence $\left\{U^{n} a\right\}_{n \in \mathbb{Z}}$ is a Riesz basis for $\mathcal{A}_{a}$ if and only if the singular part $\mu_{a}^{s} \equiv 0$ and

$$
0<\operatorname{essinf}_{\theta \in(-\pi, \pi)} \phi_{a}(\theta) \leq \operatorname{essup}_{\theta \in(-\pi, \pi)} \phi_{a}(\theta)<\infty .
$$


Theorem 2.1 is just the one-generator case $(L=1)$ of Theorem 5.1 proved below. It is worth to mention that a straightforward computation shows that the dual Riesz basis of $\left\{U^{n} a\right\}_{n \in \mathbb{Z}}$ in $\mathcal{A}_{a}$ is given by $\left\{U^{n} b\right\}_{n \in \mathbb{Z}}$ with $b=\sum_{k \in \mathbb{Z}} b_{k} U^{k} a \in \mathcal{A}_{a}$, where the terms of the sequence $\left\{b_{k}\right\}_{k \in \mathbb{Z}} \in \ell^{2}(\mathbb{Z})$ are the Fourier coefficients of the function $1 / \phi_{a}(\theta) \in L^{2}(-\pi, \pi)$. Indeed, for $b=\sum_{k \in \mathbb{Z}} b_{k} U^{k} a$ in $\mathcal{A}_{a}$, the biorthogonality between the sequences $\left\{U^{n} a\right\}_{n \in \mathbb{Z}}$ and $\left\{U^{n} b\right\}_{n \in \mathbb{Z}}$ means

$$
\begin{aligned}
\delta_{m, 0} & =\left\langle U^{m} a, b\right\rangle_{\mathcal{H}}=\left\langle U^{m} a, \sum_{k \in \mathbb{Z}} b_{k} U^{k} a\right\rangle_{\mathcal{H}}=\sum_{k \in \mathbb{Z}} \bar{b}_{k} \frac{1}{2 \pi} \int_{-\pi}^{\pi} \mathrm{e}^{i(m-k) \theta} \phi_{a}(\theta) d \theta \\
& =\frac{1}{2 \pi} \int_{-\pi}^{\pi}\left(\sum_{k \in \mathbb{Z}} \bar{b}_{k} \mathrm{e}^{-i k \theta}\right) \phi_{a}(\theta) \mathrm{e}^{i m \theta} d \theta=\frac{1}{2 \pi} \int_{-\pi}^{\pi} B(\theta) \phi_{a}(\theta) \mathrm{e}^{-i m \theta} d \theta,
\end{aligned}
$$

where $B(\theta):=\sum_{k \in \mathbb{Z}} b_{k} \mathrm{e}^{i k \theta}$; in other words, we have $B(\theta) \phi_{a}(\theta) \equiv 1$ in $L^{2}(-\pi, \pi)$. Moreover, it is easy to deduce that $\phi_{b}(\theta)=1 / \phi_{a}(\theta), \theta \in(-\pi, \pi)$; that is, for $k \in \mathbb{Z}$ we obtain $\left\langle U^{k} b, b\right\rangle_{\mathcal{H}}=\frac{1}{2 \pi} \int_{-\pi}^{\pi} \mathrm{e}^{\mathrm{i} k \theta} \frac{d \theta}{\phi_{a}(\theta)}$.

Finally, for the shift operator $T: f(u) \mapsto f(u-1)$ in $L^{2}(\mathbb{R})$, Theorem 2.1 allows to recover the classical necessary and sufficient condition for the sequence $\{\varphi(t-n)\}_{n \in \mathbb{Z}}$, where $\varphi \in L^{2}(\mathbb{R})$, to be a Riesz basis for the corresponding shiftinvariant subspace $\mathcal{A}_{\varphi}$ in $L^{2}(\mathbb{R})$. Indeed, consider the Fourier transform as $\hat{\varphi}(\theta):=$ $\frac{1}{\sqrt{2 \pi}} \int_{-\infty}^{\infty} \varphi(t) \mathrm{e}^{-i t \theta} d \theta$ in $L^{1}(\mathbb{R}) \cap L^{2}(\mathbb{R})$; using the Parseval's equality one easily gets

$$
\begin{aligned}
\left\langle T^{k} \varphi, \varphi\right\rangle_{L^{2}(\mathbb{R})} & =\int_{-\infty}^{\infty} \varphi(u-k) \overline{\varphi(u)} d u=\int_{-\infty}^{\infty} \widehat{\varphi(u-k)}(\theta) \overline{\hat{\varphi}(\theta)} d \theta \\
& =\int_{-\infty}^{\infty}|\widehat{\varphi}(\theta)|^{2} \mathrm{e}^{-i k \theta} d \theta=\int_{-\pi}^{\pi} \sum_{n \in \mathbb{Z}}|\hat{\varphi}(\theta+2 \pi n)|^{2} \mathrm{e}^{-i k \theta} d \theta \\
& =\frac{1}{2 \pi} \int_{-\pi}^{\pi} \mathrm{e}^{-i k \theta} 2 \pi \sum_{n \in \mathbb{Z}}|\widehat{\varphi}(-\theta+2 \pi n)|^{2} d \theta,
\end{aligned}
$$

that is, $\phi_{\varphi}(\theta)=2 \pi \sum_{n \in \mathbb{Z}}|\widehat{\varphi}(-\theta+2 \pi n)|^{2}, \theta \in(-\pi, \pi)$. Thus, Theorem 2.1 yields the classical condition (see, for instance, [8]):

$$
0<\underset{\theta \in(-\pi, \pi)}{\operatorname{essinf}} \sum_{n \in \mathbb{Z}}|\widehat{\varphi}(\theta+2 \pi n)|^{2} \leq \operatorname{esssup}_{\theta \in(-\pi, \pi)} \sum_{n \in \mathbb{Z}}|\widehat{\varphi}(\theta+2 \pi n)|^{2}<\infty .
$$

The following isomorphism between $L^{2}(0,1)$ and $\mathcal{A}_{a}$ will be crucial along this paper.

\section{The isomorphism $\mathcal{T}_{U, a}$}

We define the isomorphism $\mathcal{T}_{U, a}$ which maps the orthonormal basis $\left\{\mathrm{e}^{2 \pi i n w}\right\}_{n \in \mathbb{Z}}$ for $L^{2}(0,1)$ onto the Riesz basis $\left\{U^{n} a\right\}_{n \in \mathbb{Z}}$ for $\mathcal{A}_{a}$, that is,

$$
\begin{aligned}
& \mathcal{T}_{U, a}: L^{2}(0,1) \rightarrow \mathcal{A}_{a}, \\
& F=\sum_{n \in \mathbb{Z}} \alpha_{n} \mathrm{e}^{2 \pi \mathrm{i} n w} \mapsto x=\sum_{n \in \mathbb{Z}} \alpha_{n} U^{n} a .
\end{aligned}
$$


The following $U$-shift property holds: For any $F \in L^{2}(0,1)$ and $N \in \mathbb{Z}$, we have

$$
\mathcal{T}_{U, a}\left(F \mathrm{e}^{2 \pi \mathrm{i} N w}\right)=U^{N}\left(\mathcal{T}_{U, a} F\right) .
$$

\section{The U-systems}

For any fixed $b \in \mathcal{H}$ we define the $U$-system $\mathcal{L}_{b}$ as the linear operator between $\mathcal{H}$ and the set $C(\mathbb{R})$ of the continuous functions on $\mathbb{R}$ given by

$$
\mathcal{H} \ni x \mapsto \mathcal{L}_{b} x \in C(\mathbb{R}) \text { such that } \mathcal{L}_{b} x(t):=\left\langle x, U^{t} b\right\rangle_{\mathcal{H}}, t \in \mathbb{R} .
$$

For any $x \in \mathcal{A}_{a}$ and $t \in \mathbb{R}$, by using the Plancherel equality for the orthonormal basis $\left\{\mathrm{e}^{2 \pi \mathrm{in} w}\right\}_{n \in \mathbb{Z}}$ in $L^{2}(0,1)$, we have

$$
\begin{aligned}
\mathcal{L}_{b} x(t) & =\left\langle x, U^{t} b\right\rangle_{\mathcal{H}}=\left\langle\sum_{n \in \mathbb{Z}} \alpha_{n} U^{n} a, U^{t} b\right\rangle_{\mathcal{H}}=\sum_{n \in \mathbb{Z}} \alpha_{n}{\overline{\left\langle U^{t} b, U^{n} a\right\rangle_{\mathcal{H}}}}=\left\langle F, \sum_{n \in \mathbb{Z}}\left\langle U^{t} b, U^{n} a\right\rangle_{\mathcal{H}} \mathrm{e}^{2 \pi \mathrm{in} w}\right\rangle_{L^{2}(0,1)}=\left\langle F, K_{t}\right\rangle_{L^{2}(0,1)},
\end{aligned}
$$

where $\mathcal{T}_{U, a} F=x$, and the function

$$
K_{t}(w):=\sum_{n \in \mathbb{Z}}\left\langle U^{t} b, U^{n} a\right\rangle_{\mathcal{H}} \mathrm{e}^{2 \pi \mathrm{i} n w}=\sum_{n \in \mathbb{Z}} \overline{\mathcal{L}_{b} a(t-n)} \mathrm{e}^{2 \pi \mathrm{i} n w}
$$

belongs to $L^{2}(0,1)$ since the sequence $\left\{\left\langle U^{t} b, U^{n} a\right\rangle_{\mathcal{H}}\right\}_{n \in \mathbb{Z}}$ belongs to $\ell^{2}(\mathbb{Z})$ for each $t \in \mathbb{R}$.

\section{An expression for the generalized samples}

Suppose that $s$ vectors $b_{j} \in \mathcal{H}, j=1,2, \ldots, s$, are given and consider their associated $U$-systems $\mathcal{L}_{j}:=\mathcal{L}_{b_{j}}, j=1,2, \ldots, s$. Our aim is the stable recovery of any $x \in \mathcal{A}_{a}$ from the sequence of samples $\left\{\mathcal{L}_{j} x(\mathrm{rm})\right\}_{m \in \mathbb{Z} ; j=1,2, \ldots, s}$ where $r \geq 1$. To

this end, first we obtain a suitable expression for the samples. For $x \in \mathcal{A}_{a}$ let $F \in L^{2}(0,1)$ such that $\mathcal{T}_{U, a} F=x$; by using $(2.2)$, for $j=1,2, \ldots, s$ and $m \in \mathbb{Z}$ we have

$$
\begin{aligned}
\mathcal{L}_{j} x(r m) & =\left\langle F, \sum_{n \in \mathbb{Z}}\left\langle U^{r m} b_{j}, U^{n} a\right\rangle_{\mathcal{H}} \mathrm{e}^{2 \pi \mathrm{i} n w}\right\rangle_{L^{2}(0,1)} \\
& =\left\langle F, \sum_{k \in \mathbb{Z}}\left\langle U^{k} b_{j}, a\right\rangle_{\mathcal{H}} \mathrm{e}^{2 \pi \mathrm{i}(r m-k) w}\right\rangle_{L^{2}(0,1)} \\
& =\left\langle F,\left[\sum_{k \in \mathbb{Z}} \overline{\left\langle a, U^{k} b_{j}\right\rangle_{\mathcal{H}}} \mathrm{e}^{-2 \pi \mathrm{i} k w}\right] \mathrm{e}^{2 \pi \mathrm{i} r m w}\right\rangle_{L^{2}(0,1)},
\end{aligned}
$$

where the change in the summation's index $k:=r m-n$ has been done. Hence,

$$
\mathcal{L}_{j} x(r m)=\left\langle F, \overline{g_{j}(w)} \mathrm{e}^{2 \pi \mathrm{irmw}}\right\rangle_{L^{2}(0,1)} \text { for } m \in \mathbb{Z} \text { and } j=1,2, \ldots, s,
$$


where the function

$$
g_{j}(w):=\sum_{k \in \mathbb{Z}} \mathcal{L}_{j} a(k) \mathrm{e}^{2 \pi \mathrm{i} k w}
$$

belongs to $L^{2}(0,1)$ for each $j=1,2, \ldots, s$.

As a consequence of (2.3), the stable recovery of any $x \in \mathcal{A}_{a}$ depends on whether the sequence $\left\{\overline{g_{j}(w)} \mathrm{e}^{2 \pi i r m w}\right\}_{m \in \mathbb{Z} ; j=1,2, \ldots, s}$ forms a frame for $L^{2}(0,1)$. Recall that a sequence $\left\{x_{n}\right\}_{n \in \mathbb{Z}}$ is a frame for a separable Hilbert space $\mathcal{H}$ if there exist two constants $A, B>0$ (frame bounds) such that

$$
A\|x\|^{2} \leq \sum_{n \in \mathbb{Z}}\left|\left\langle x, x_{n}\right\rangle\right|^{2} \leq B\|x\|^{2} \quad \text { for all } x \in \mathcal{H}
$$

A sequence $\left\{x_{n}\right\}_{n \in \mathbb{Z}}$ in $\mathcal{H}$ satisfying only the right-hand side inequality above is said to be a Bessel sequence for $\mathcal{H}$. Given a frame $\left\{x_{n}\right\}_{n \in \mathbb{Z}}$ for $\mathcal{H}$ the representation property of any vector $x \in \mathcal{H}$ as a series $x=\sum_{n \in \mathbb{Z}} c_{n} x_{n}$ is retained, but, unlike the case of Riesz bases (exact frames), the uniqueness of this representation (for overcomplete frames) is sacrificed. Suitable frame coefficients $c_{n}$ which depend continuously and linearly on $x$ are obtained by using the dual frames $\left\{y_{n}\right\}_{n \in \mathbb{Z}}$ of $\left\{x_{n}\right\}_{n \in \mathbb{Z}}$, i.e. $\left\{y_{n}\right\}_{n \in \mathbb{Z}}$ is another frame for $\mathcal{H}$ such that $x=\sum_{n \in \mathbb{Z}}\left\langle x, y_{n}\right\rangle x_{n}=\sum_{n \in \mathbb{Z}}\left\langle x, x_{n}\right\rangle y_{n}$ for each $x \in \mathcal{H}$. For more details on frame theory see [8].

A deep study of sequences having the form of $\left\{\overline{g_{j}(w)} \mathrm{e}^{2 \pi \mathrm{i} r m w}\right\}_{m \in \mathbb{Z} ; j=1,2, \ldots, s}$ was done in $[13,14]$. Namely, consider the $s \times r$ matrix of functions in $L^{2}(0,1)$

$$
\begin{aligned}
\mathbb{G}(w) & :=\left[\begin{array}{cccc}
g_{1}(w) & g_{1}\left(w+\frac{1}{r}\right) & \cdots & g_{1}\left(w+\frac{r-1}{r}\right) \\
g_{2}(w) & g_{2}\left(w+\frac{1}{r}\right) & \cdots & g_{2}\left(w+\frac{r-1}{r}\right) \\
\vdots & \vdots & \\
g_{s}(w) & g_{s}\left(w+\frac{1}{r}\right) & \cdots & g_{s}\left(w+\frac{r-1}{r}\right)
\end{array}\right] \\
= & {\left[\begin{array}{lll}
\left.g_{j}\left(w+\frac{k-1}{r}\right)\right]_{\substack{j=1,2, \ldots, s \\
k=1,2, \ldots, r}}
\end{array}\right.}
\end{aligned}
$$

and its related constants

$$
\alpha_{\mathbb{G}}:=\operatorname{essinf}_{w \in(0,1 / r)} \lambda_{\min }\left[\mathbb{G}^{*}(w) \mathbb{G}(w)\right], \quad \beta_{\mathbb{G}}:=\underset{w \in(0,1 / r)}{\operatorname{essiup}} \lambda_{\max }\left[\mathbb{G}^{*}(w) \mathbb{G}(w)\right]
$$

where $\mathbb{G}^{*}(w)$ denotes the transpose conjugate of the matrix $\mathbb{G}(w)$, and $\lambda_{\min }$ (respectively, $\lambda_{\max }$ ) the smallest (respectively, the largest) eigenvalue of the positive semidefinite matrix $\mathbb{G}^{*}(w) \mathbb{G}(w)$. Observe that $0 \leq \alpha_{\mathbb{G}} \leq \beta_{\mathbb{G}} \leq \infty$. Notice that in the definition of the matrix $\mathbb{G}(w)$ we are considering 1-periodic extensions of the involved functions $g_{j}, j=1,2, \ldots, s$. 
A complete characterization of the sequence $\left\{\overline{g_{j}(w)} \mathrm{e}^{2 \pi \mathrm{i} r m w}\right\}_{m \in \mathbb{Z} ; j=1,2, \ldots, s}$ is given in the next lemma (see [14, Lemma 3] or [13, Lemma 2] for the proof).

Lemma 2.2. For the functions $g_{j} \in L^{2}(0,1), j=1,2, \ldots, s$, consider the associated matrix $\mathbb{G}(w)$ given in (2.5). Then, the following results hold:

(a) The sequence $\left\{\overline{g_{j}(w)} \mathrm{e}^{2 \pi \mathrm{irnw}}\right\}_{n \in \mathbb{Z} ; j=1,2, \ldots, s}$ is a complete system for $L^{2}(0,1)$ if and only if the rank of the matrix $\mathbb{G}(w)$ is $r$ a.e. in $(0,1 / r)$.

(b) The sequence $\left\{\overline{g_{j}(w)} \mathrm{e}^{2 \pi \mathrm{irnw}}\right\}_{n \in \mathbb{Z} ; j=1,2, \ldots, s}$ is a Bessel sequence for $L^{2}(0,1)$ if and only if $g_{j} \in L^{\infty}(0,1)$ (or equivalently $\left.\beta_{\mathbb{G}}<\infty\right)$. In this case, the optimal Bessel bound is $\beta_{\mathbb{G}} / r$.

(c) The sequence $\left\{\overline{g_{j}(w)} \mathrm{e}^{2 \pi \mathrm{i} r n w}\right\}_{n \in \mathbb{Z} ; j=1,2, \ldots, s}$ is a frame for $L^{2}(0,1)$ if and only if $0<\alpha_{\mathbb{G}} \leq \beta_{\mathbb{G}}<\infty$. In this case, the optimal frame bounds are $\alpha_{\mathbb{G}} / r$ and $\beta_{\mathbb{G}} / r$.

(d) The sequence $\left\{\overline{g_{j}(w)} \mathrm{e}^{2 \pi \mathrm{i} n w w}\right\}_{n \in \mathbb{Z} ; j=1,2, \ldots, s}$ is a Riesz basis for $L^{2}(0,1)$ if and only if is a frame and $s=r$.

A comment about Lemma 2.2 in terms of the average sampling terminology introduced by Aldroubi et al. in [6] is in order. According to [6], we say that:

(1) The set $\left\{\mathcal{L}_{1}, \mathcal{L}_{2}, \ldots, \mathcal{L}_{s}\right\}$ is an $r$-determining $U$-sampler for $\mathcal{A}_{a}$ if the only vector $x \in \mathcal{A}_{a}$, satisfying $\mathcal{L}_{j} x(r m)=0$ for all $j=1,2, \ldots, s$ and $m \in \mathbb{Z}$, is $x=0$.

(2) The set $\left\{\mathcal{L}_{1}, \mathcal{L}_{2}, \ldots, \mathcal{L}_{s}\right\}$ is an $r$-stable $U$-sampler for $\mathcal{A}_{a}$ if there exist positive constants $A$ and $B$ such that

$$
A\|x\|^{2} \leq \sum_{j=1}^{s} \sum_{m \in \mathbb{Z}}\left|\mathcal{L}_{j} x(r m)\right|^{2} \leq B\|x\|^{2} \quad \text { for all } x \in \mathcal{A}_{a} .
$$

Hence, parts (a) and (c) of Lemma 2.2 can be read, by using (2.3), as follows:

(i) The set $\left\{\mathcal{L}_{1}, \mathcal{L}_{2}, \ldots, \mathcal{L}_{s}\right\}$ is an $r$-determining $U$-sampler for $\mathcal{A}_{a}$ if and only if $\operatorname{rank} \mathbb{G}(w)=r$ a.e. in $(0,1)$ (and hence, necessarily, $s \geq r$ ).

(ii) The set $\left\{\mathcal{L}_{1}, \mathcal{L}_{2}, \ldots, \mathcal{L}_{s}\right\}$ is an $r$-stable $U$-sampler for $\mathcal{A}_{a}$ if and only if $0<$ $\alpha_{\mathbb{G}} \leq \beta_{\mathbb{G}}<\infty$.

An $r$-determining $U$-sampler for $\mathcal{A}_{a}$ can distinguish between two distinct elements in $\mathcal{A}_{a}$, but the recovery, if any, is not necessarily stable. If the system $\left\{\mathcal{L}_{1}, \mathcal{L}_{2}, \ldots, \mathcal{L}_{s}\right\}$ is an $r$-stable $U$-sampler for $\mathcal{A}_{a}$, then any $x \in \mathcal{A}_{a}$ can be recovered, in a stable way, from the sequence of generalized samples $\left\{\mathcal{L}_{j} x(r m)\right\}_{m \in \mathbb{Z} ; j=1,2, \ldots, s}$, where necessarily $s \geq r$. Roughly speaking, the operator which maps

$$
\mathcal{A}_{a} \ni x \mapsto\left\{\mathcal{L}_{j} x(r m)\right\}_{m \in \mathbb{Z} ; j=1,2, \ldots, s} \in \ell_{s}^{2}(\mathbb{Z}):=\ell^{2}(\mathbb{Z}) \underset{(s \text { times })}{\times \cdots \times} \ell^{2}(\mathbb{Z})
$$

has a bounded inverse.

Having in mind (2.3), from the sequence of samples $\left\{\mathcal{L}_{j} x(\mathrm{rm})\right\}_{m \in \mathbb{Z} ; j=1,2, \ldots, s}$ we recover $F \in L^{2}(0,1)$, and by means of the isomorphism $\mathcal{T}_{U, a}$, the vector $x=\mathcal{T}_{U, a} F$ in $\mathcal{A}_{a}$. This will be the main goal in the next section. 


\section{Generalized Regular Sampling in $\mathcal{A}_{a}$}

Along with the characterization of the sequence $\left\{\overline{g_{j}(w)} \mathrm{e}^{2 \pi \mathrm{i} n n w}\right\}_{n \in \mathbb{Z} ; j=1,2, \ldots, s}$ as a frame in $L^{2}(0,1)$, in [14] a family of dual frames are also given: Choose functions $h_{j}$ in $L^{\infty}(0,1), j=1,2, \ldots, s$, such that

$$
\left[h_{1}(w), h_{2}(w), \ldots, h_{s}(w)\right] \mathbb{G}(w)=[1,0, \ldots, 0] \text { a.e. in }(0,1) .
$$

It was proven in $[14]$ that the sequence $\left\{r h_{j}(w) \mathrm{e}^{2 \pi i r n w}\right\}_{n \in \mathbb{Z} ; j=1,2, \ldots, s}$ is a dual frame of the sequence $\left\{\overline{g_{j}(w)} \mathrm{e}^{2 \pi \mathrm{i} r n w}\right\}_{n \in \mathbb{Z} ; j=1,2, \ldots, s}$ in $L^{2}(0,1)$. In other words, taking into account (2.3), we have for any $F \in L^{2}(0,1)$ the expansion

$$
F=\sum_{j=1}^{s} \sum_{m \in \mathbb{Z}} \mathcal{L}_{j} x(r m) r h_{j}(w) \mathrm{e}^{2 \pi \mathrm{i} r m w} \quad \text { in } L^{2}(0,1) .
$$

Concerning to the existence of the functions $h_{j}, j=1,2, \ldots, s$, consider the first row of the $r \times s$ Moore-Penrose pseudo-inverse $\mathbb{G}^{\dagger}(w)$ of $\mathbb{G}(w)$ given by

$$
\mathbb{G}^{\dagger}(w):=\left[\mathbb{G}^{*}(w) \mathbb{G}(w)\right]^{-1} \mathbb{G}^{*}(w) .
$$

Its entries are essentially bounded in $(0,1)$ since the functions $g_{j}, j=1,2, \ldots, s$, and $\operatorname{det}^{-1}\left[\mathbb{G}^{*}(w) \mathbb{G}(w)\right]$ are essentially bounded in $(0,1)$, and $(3.1)$ trivially holds. All the possible solutions of (3.1) are given by the first row of the $r \times s$ matrices given by

$$
\mathbb{H}_{\mathbb{K}}(w):=\mathbb{G}^{\dagger}(w)+\mathbb{K}(w)\left[\mathbb{I}_{s}-\mathbb{G}(w) \mathbb{G}^{\dagger}(w)\right]
$$

where $\mathbb{K}(w)$ denotes any $r \times s$ matrix with entries in $L^{\infty}(0,1)$, and $\mathbb{I}_{s}$ is the identity matrix of order $s$.

Applying the isomorphism $\mathcal{T}_{U, a}$ in $(3.2)$, for $x=\mathcal{T}_{U, a} F \in \mathcal{A}_{a}$ we obtain the sampling expansion:

$$
\begin{aligned}
x & =\sum_{j=1}^{s} \sum_{m \in \mathbb{Z}} \mathcal{L}_{j} x(r m) \mathcal{T}_{U, a}\left[r h_{j}(\cdot) \mathrm{e}^{2 \pi \mathrm{i} r m \cdot}\right]=\sum_{j=1}^{s} \sum_{m \in \mathbb{Z}} \mathcal{L}_{j} x(r m) U^{r m}\left[\mathcal{T}_{U, a}\left(r h_{j}\right)\right] \\
& =\sum_{j=1}^{s} \sum_{m \in \mathbb{Z}} \mathcal{L}_{j} x(r m) U^{r m} c_{j, h} \quad \text { in } \mathcal{H},
\end{aligned}
$$

where $c_{j, h}:=\mathcal{T}_{U, a}\left(r h_{j}\right) \in \mathcal{A}_{a}, j=1,2, \ldots, s$, and we have used the $U$-shift property (2.1). Besides, the sequence $\left\{U^{r m} c_{j, h}\right\}_{m \in \mathbb{Z} ; j=1,2, \ldots, s}$ is a frame for $\mathcal{A}_{a}$. In fact, the following result holds.

Theorem 3.1. Let $b_{j} \in \mathcal{H}$ and let $\mathcal{L}_{j}$ be its associated $U$-system for $j=1,2, \ldots, s$. Assume that the function $g_{j}, j=1,2, \ldots, s$, given in $(2.4)$ belongs to $L^{\infty}(0,1)$; or equivalently, that $\beta_{\mathbb{G}}<\infty$ for the associated $s \times r$ matrix $\mathbb{G}(w)$. The following statements are equivalent:

(a) $\alpha_{\mathbb{G}}>0$. 
(b) There exists a vector $\left[h_{1}(w), h_{2}(w), \ldots, h_{s}(w)\right]$ with entries in $L^{\infty}(0,1)$ satisfying

$$
\left[h_{1}(w), h_{2}(w), \ldots, h_{s}(w)\right] \mathbb{G}(w)=[1,0, \ldots, 0] \text { a.e. in }(0,1)
$$

(c) There exist elements $c_{j} \in \mathcal{A}_{a}, j=1,2, \ldots, s$, such that the sequence $\left\{U^{r k} c_{j}\right\}_{k \in \mathbb{Z} ; j=1,2, \ldots, s}$ is a frame for $\mathcal{A}_{a}$, and for any $x \in \mathcal{A}_{a}$ the expansion

$$
x=\sum_{j=1}^{s} \sum_{k \in \mathbb{Z}} \mathcal{L}_{j} x(r k) U^{r k} c_{j} \quad \text { in } \mathcal{H}
$$

holds.

(d) There exists a frame $\left\{C_{j, k}\right\}_{k \in \mathbb{Z} ; j=1,2, \ldots, s}$ for $\mathcal{A}_{a}$ such that, for each $x \in \mathcal{A}_{a}$ the expansion

$$
x=\sum_{j=1}^{s} \sum_{k \in \mathbb{Z}} \mathcal{L}_{j} x(r k) C_{j, k} \quad \text { in } \mathcal{H}
$$

holds.

Proof. We have already proved that (a) implies (b) and that (b) implies (c). Obviously, (c) implies (d). As a consequence, we only need to prove that (d) implies (a). Applying the isomorphism $\mathcal{T}_{U, a}^{-1}$ to the expansion in (d), and taking into account (2.3) we obtain

$$
\begin{aligned}
F & =\mathcal{T}_{U, a}^{-1} x=\sum_{j=1}^{s} \sum_{k \in \mathbb{Z}} \mathcal{L}_{j} x(r k) \mathcal{T}_{U, a}^{-1}\left(C_{j, k}\right) \\
& =\sum_{j=1}^{s} \sum_{k \in \mathbb{Z}}\left\langle F, \overline{g_{j}(w)} \mathrm{e}^{2 \pi i r k w}\right\rangle_{L^{2}(0,1)} \mathcal{T}_{U, a}^{-1}\left(C_{j, k}\right) \text { in } L^{2}(0,1),
\end{aligned}
$$

where the sequence $\left\{\mathcal{T}_{U, a}^{-1}\left(C_{j, k}\right)\right\}_{k \in \mathbb{Z} ; j=1,2, \ldots, s}$ is a frame for $L^{2}(0,1)$. The sequence $\left\{\overline{g_{j}(w)} \mathrm{e}^{2 \pi \mathrm{i} r m w}\right\}_{m \in \mathbb{Z} ; j=1,2, \ldots, s}$ is a Bessel sequence in $L^{2}(0,1)$ since $\beta_{\mathbb{G}}<\infty$, and satisfying the above expansion in $L^{2}(0,1)$. According to [8, Lemma 5.6.2], the sequences $\left\{\mathcal{T}_{U, a}^{-1}\left(C_{j, k}\right)\right\}_{k \in \mathbb{Z} ; j=1,2, \ldots, s}$ and $\left\{\overline{g_{j}(w)} \mathrm{e}^{2 \pi \mathrm{i} r k w}\right\}_{k \in \mathbb{Z} ; j=1,2, \ldots, s}$ form a pair of dual frames in $L^{2}(0,1)$; in particular, by using Lemma 2.2 we obtain that $\alpha_{\mathbb{G}}>0$ which concludes the proof.

In case the functions $g_{j}, j=1,2, \ldots, s$ are continuous on $\mathbb{R}$, condition (a) in Theorem 3.1 can be expressed in terms of the rank of the matrix $\mathbb{G}(w)$; notice that this occurs, for example, whenever the sequences $\left\{\mathcal{L}_{j} a(k)\right\}_{k \in \mathbb{Z}}, j=1,2, \ldots, s$, belong to $\ell^{1}(\mathbb{Z})$.

Corollary 3.2. Assume that the 1-periodic extension of the functions $g_{j}, j=$ $1,2, \ldots, s$, given in (2.4) are continuous on $\mathbb{R}$. Then, the following conditions are 
equivalent:

(i) $\operatorname{rank} \mathbb{G}(w)=r$ for all $w \in \mathbb{R}$.

(ii) There exist $c_{j} \in \mathcal{A}_{a}, j=1,2, \ldots, s$, such that the sequence $\left\{U^{r k} c_{j}\right\}_{k \in \mathbb{Z} ; j=1,2, \ldots, s}$ is a frame for $\mathcal{A}_{a}$, and the sampling formula (3.5) holds for each $x \in \mathcal{A}_{a}$.

Proof. Whenever the functions $g_{j}, j=1,2, \ldots, s$, are continuous on $\mathbb{R}$, the condition $\alpha_{\mathbb{G}}>0$ is equivalent to $\operatorname{det}\left[\mathbb{G}^{*}(w) \mathbb{G}(w)\right] \neq 0$ for all $w \in \mathbb{R}$. Indeed, if $\operatorname{det} \mathbb{G}^{*}(w) \times$ $\mathbb{G}(w)>0$ then the first row of the matrix $\mathbb{G}^{\dagger}(w):=\left[\mathbb{G}^{*}(w) \mathbb{G}(w)\right]^{-1} \mathbb{G}^{*}(w)$, gives a vector $\left[h_{1}, h_{2}, \ldots, h_{s}\right]$ satisfying the statement (b) in Theorem 3.1 and, as a consequence, $\alpha_{\mathbb{G}}>0$. The converse follows from the fact that $\operatorname{det}\left[\mathbb{G}^{*}(w) \mathbb{G}(w)\right] \geq \alpha_{\mathbb{G}}^{r}$ for all $w \in \mathbb{R}$. Since, $\operatorname{det}\left[\mathbb{G}^{*}(w) \mathbb{G}(w)\right] \neq 0$ is equivalent to $\operatorname{rank} \mathbb{G}(w)=r$ for all $w \in \mathbb{R}$, the result is a consequence of Theorem 3.1 .

Whenever the sampling period $r$ equals the number of $U$-systems $s$ we are in the presence of Riesz bases, and there exists a unique sampling expansion in Theorem 3.1.

Corollary 3.3. Let $b_{j} \in \mathcal{H}$ for $j=1,2, \ldots, r$, i.e. $r=s$ in Theorem 3.1. Let $\mathcal{L}_{j}$ be its associated $U$-system for $j=1,2, \ldots, r$. Assume that the function $g_{j}$, $j=1,2, \ldots, r$, given in (2.4) belongs to $L^{\infty}(0,1)$; or equivalently, $\beta_{\mathbb{G}}<\infty$ for the associated $r \times r$ matrix $\mathbb{G}(w)$. The following statements are equivalent:

(a) $\alpha_{\mathbb{G}}>0$.

(b) There exists a Riesz basis $\left\{C_{j, k}\right\}_{k \in \mathbb{Z} ; j=1,2, \ldots, r}$ such that for any $x \in \mathcal{A}_{a}$ the expansion

$$
x=\sum_{j=1}^{r} \sum_{k \in \mathbb{Z}} \mathcal{L}_{j} x(r k) C_{j, k} \quad \text { in } \mathcal{H}
$$

holds.

In case the equivalent conditions are satisfied, necessarily there exist $c_{j} \in \mathcal{A}_{a}, j=$ $1,2, \ldots, r$, such that $C_{j, k}=U^{r k} c_{j}$ for $k \in \mathbb{Z}$ and $j=1,2, \ldots, r$. Moreover, the interpolation property $\mathcal{L}_{j^{\prime}} c_{j}(r k)=\delta_{j, j^{\prime}} \delta_{k, 0}$, where $k \in \mathbb{Z}$ and $j, j^{\prime}=1,2, \ldots, r$, holds.

Proof. Assume that $\alpha_{\mathbb{G}}>0$; since $\mathbb{G}(w)$ is a square matrix, this implies that

$$
\underset{w \in \mathbb{R}}{\operatorname{essinf}}|\operatorname{det} \mathbb{G}(w)|>0 .
$$

Thus, the unique solution $\left[h_{1}(w), h_{2}(w), \ldots, h_{r}(w)\right]$ of $(3.1)$ with $h_{j} \in L^{\infty}(0,1)$ for $j=1,2, \ldots, r$ is given by the first row of the matrix $\mathbb{G}^{-1}(w)$. According to Theorem 3.1, the sequence $\left\{C_{j, k}\right\}_{k \in \mathbb{Z} ; j=1,2, \ldots, r}:=\left\{U^{r k} c_{j}\right\}_{k \in \mathbb{Z} ; j=1,2, \ldots, r}$, where $c_{j}=\mathcal{T}_{U, a}\left(r h_{j}\right)$, satisfies the sampling formula (3.6). Moreover, the sequence $\left\{r h_{j}(w) \mathrm{e}^{2 \pi i r k w}\right\}_{k \in \mathbb{Z} ; j=1,2, \ldots, r}=\left\{\mathcal{T}_{U, a}^{-1}\left(U^{r k} c_{j}\right)\right\}_{k \in \mathbb{Z} ; j=1,2, \ldots, r}$ is a frame for $L^{2}(0,1)$. 
Since $r=s$, according to Lemma 2.2, it is a Riesz basis. Hence, $\left\{U^{r k} c_{j}\right\}_{k \in \mathbb{Z} ; j=1,2, \ldots, r}$ is a Riesz basis for $\mathcal{A}_{a}$ and (b) is proved.

Conversely, assume now that $\left\{C_{j, k}\right\}_{k \in \mathbb{Z} ; j=1,2, \ldots, r}$ is a Riesz basis for $\mathcal{A}_{a}$ satisfying (3.6). From the uniqueness of the coefficients in a Riesz basis, we get that the interpolatory condition $\left(\mathcal{L}_{j^{\prime}} C_{j, k}\right)\left(r k^{\prime}\right)=\delta_{j, j^{\prime}} \delta_{k, k^{\prime}}$ holds for $j, j^{\prime}=1,2, \ldots, r$ and $k, k^{\prime} \in \mathbb{Z}$. Since $\mathcal{T}_{U, a}^{-1}$ is an isomorphism, the sequence $\left\{\mathcal{T}_{U, a}^{-1}\left(C_{j, k}\right)\right\}_{k \in \mathbb{Z} ; j=1,2, \ldots, r}$ is a Riesz basis for $L^{2}(0,1)$. Expanding the function $\overline{g_{j^{\prime}}(w)} \mathrm{e}^{-2 \pi \mathrm{ir} k^{\prime} w}$ with respect to the dual basis of $\left\{\mathcal{T}_{U, a}^{-1}\left(C_{j, k}\right)\right\}_{k \in \mathbb{Z} ; j=1,2, \ldots, r}$, denoted by $\left\{D_{j, k}\right\}_{k \in \mathbb{Z} ; j=1,2, \ldots, r}$, and having in mind (2.3) we obtain

$$
\begin{aligned}
\overline{g_{j^{\prime}}(w)} \mathrm{e}^{2 \pi \mathrm{i} r k^{\prime} w} & =\sum_{j=1}^{r} \sum_{k \in \mathbb{Z}}\left\langle\overline{\left\langle g_{j^{\prime}}(\cdot)\right.} \mathrm{e}^{2 \pi \mathrm{i} r k^{\prime} \cdot}, \mathcal{T}_{U, a}^{-1}\left(C_{j, k}\right)\right\rangle_{L^{2}(0,1)} D_{j, k}(w) \\
& =\sum_{k \in \mathbb{Z}} \overline{\mathcal{L}_{j^{\prime}} C_{j, k}\left(r k^{\prime}\right)} D_{j, k}(w)=D_{j^{\prime}, k^{\prime}}(w) .
\end{aligned}
$$

Therefore, the sequence $\left\{\overline{g_{j}(w)} \mathrm{e}^{2 \pi \mathrm{irkw}}\right\}_{k \in \mathbb{Z} ; j=1,2, \ldots, r}$ is the dual basis of the Riesz basis $\left\{\mathcal{T}_{U, a}^{-1}\left(C_{j, k}\right)\right\}_{k \in \mathbb{Z} ; j=1,2, \ldots, r}$. In particular, it is a Riesz basis for $L^{2}(0,1)$, which implies, according to Lemma 2.2, that $\alpha_{\mathbb{G}}>0$, i.e. condition (a). Moreover, the sequence $\left\{\mathcal{T}_{U, a}^{-1}\left(C_{j, k}\right)\right\}_{k \in \mathbb{Z} ; j=1,2, \ldots, r}$ is necessarily the unique dual basis of the Riesz basis $\left\{\overline{g_{j}(w)} \mathrm{e}^{2 \pi \mathrm{irkw}}\right\}_{k \in \mathbb{Z} ; j=1,2, \ldots, r}$. Therefore, this proves the uniqueness of the Riesz basis $\left\{C_{j, k}\right\}_{k \in \mathbb{Z} ; j=1,2, \ldots, r}$ for $\mathcal{A}_{a}$ satisfying (3.6).

\section{Some comments on the sequence $\left\{U^{r k} b_{j}\right\}_{k \in \mathbb{Z} ; j=1,2, \ldots, s}$}

Concerning Theorem 3.1, more can be said about the sequence $\left\{U^{r k} b_{j}\right\}_{k \in \mathbb{Z} ; j=1,2, \ldots, s}$, where the vectors $b_{j} \in \mathcal{H}$ define the $U$-systems $\mathcal{L}_{j} \equiv \mathcal{L}_{b_{j}}, j=1,2, \ldots, s$. Having in mind (2.3) and the isomorphism $\mathcal{T}_{U, a}$, we obtain that

$$
\begin{aligned}
\frac{\alpha_{\mathbb{G}}}{r}\left\|\mathcal{T}_{U, a}\right\|^{-2}\|x\|^{2} & \leq \sum_{j=1}^{s} \sum_{k \in \mathbb{Z}}\left|\left\langle x, U^{r k} b_{j}\right\rangle\right|^{2} \\
& \leq \frac{\beta_{\mathbb{G}}}{r}\left\|\mathcal{T}_{U, a}^{-1}\right\|^{2}\|x\|^{2} \quad \text { for all } x \in \mathcal{A}_{a} .
\end{aligned}
$$

- In case that $b_{j} \in \mathcal{A}_{a}$ for each $j=1,2, \ldots, s$, we derive that $\left\{U^{r k} b_{j}\right\}_{k \in \mathbb{Z} ; j=1,2, \ldots, s}$ is a frame for $\mathcal{A}_{a}$, and it is dual to the frame $\left\{U^{r k} c_{j}\right\}_{k \in \mathbb{Z} ; j=1,2, \ldots, s}$ in $\mathcal{A}_{a}$. Thus, the sampling expansion (3.5) is nothing but a frame expansion in $\mathcal{A}_{a}$.

- In case that some $b_{j} \notin \mathcal{A}_{a}$, the sequence $\left\{U^{r k} b_{j}\right\}_{k \in \mathbb{Z} ; j=1,2, \ldots, s}$ is not contained in $\mathcal{A}_{a}$. However, inequalities (3.7) hold. Therefore, the sequence $\left\{U^{r k} b_{j}\right\}_{k \in \mathbb{Z} ; j=1,2, \ldots, s}$ is a pseudo-dual frame for the frame $\left\{U^{r k} c_{j}\right\}_{k \in \mathbb{Z} ; j=1,2, \ldots, s}$ in $\mathcal{A}_{a}$ (see $[20,21]$ ). Denoting by $P_{\mathcal{A}_{a}}$ the orthogonal projection onto $\mathcal{A}_{a}$, we derive from (3.7) that the sequence $\left\{P_{\mathcal{A}_{a}}\left(U^{r k} b_{j}\right)\right\}_{k \in \mathbb{Z} ; j=1,2, \ldots, s}$ is a dual frame of $\left\{U^{r k} c_{j}\right\}_{k \in \mathbb{Z} ; j=1,2, \ldots, s}$ in $\mathcal{A}_{a}$.

- Whenever $r=s$, according to the above cases, the sequence $\left\{U^{r k} b_{j}\right\}_{k \in \mathbb{Z} ; j=1,2, \ldots, s}$ is a Riesz basis or a pseudo-Riesz basis for $\mathcal{A}_{a}$. 


\section{Sampling formulas with prescribed properties}

The sampling formula (3.5) can be thought as a filter-bank. Indeed, assume that for $j=1,2, \ldots, s$ we have

$$
c_{j, h}=\mathcal{T}_{U, a}\left(r h_{j}\right)=r \sum_{n \in \mathbb{Z}} \widehat{h}_{j}(n) U^{n} a \quad \text { where } \widehat{h}_{j}(n)=\int_{0}^{1} h_{j}(w) \mathrm{e}^{-2 \pi \mathrm{i} n w} d w, \quad n \in \mathbb{Z} .
$$

Substituting in (3.5), after the change of summation index $m:=r k+n$ we obtain

$$
x=\sum_{m \in \mathbb{Z}}\left\{\sum_{j=1}^{s} \sum_{k \in \mathbb{Z}} r \mathcal{L}_{j} x(r k) \widehat{h}_{j}(m-r k)\right\} U^{m} a,
$$

that is, the relevant data is the output of a filter-bank:

$$
\alpha_{m}:=\sum_{j=1}^{s} \sum_{k \in \mathbb{Z}} r \mathcal{L}_{j} x(r k) \widehat{h}_{j}(m-r k), \quad m \in \mathbb{Z}
$$

where the input is the given samples and the impulse responses depend on the sampling vectors $c_{j, h}, j=1,2, \ldots, s$. In the oversampling setting, i.e. $s>r$, according to (3.3) there exist infinitely many sampling vectors $c_{j, h}, j=1,2, \ldots, s$, for which the sampling formula (3.5) holds. A natural question is whether we can choose the sampling vectors $c_{j, h}, j=1,2, \ldots, s$, with prescribed properties.

For instance, a challenging problem is to ask under what conditions we are in the presence of a finite impulse response filter-bank; i.e. $c_{j, h}=r \sum_{\text {finite }} \widehat{h}_{j}(n) U^{n} a$, $j=1,2, \ldots, s$, or equivalently, when the functions $h_{j}, j=1, \ldots, s$, are $2 \pi$-periodic trigonometric polynomials. Instead, we deal with Laurent polynomials by using the variable $z=\mathrm{e}^{2 \pi \mathrm{i} w}$, that is, $\mathrm{g}_{j}(z):=\sum_{k \in \mathbb{Z}} \mathcal{L}_{j} a(k) z^{k}, j=1,2, \ldots, s$. We introduce the $s \times r$ matrix

$$
\mathrm{G}(z):=\left[\begin{array}{cccc}
\mathrm{g}_{1}(z) & \mathrm{g}_{1}(z W) & \cdots & \mathrm{g}_{1}\left(z W^{r-1}\right) \\
\mathrm{g}_{2}(z) & \mathrm{g}_{2}(z W) & \cdots & \mathrm{g}_{2}\left(z W^{r-1}\right) \\
\vdots & \vdots & & \vdots \\
\mathrm{g}_{s}(z) & \mathrm{g}_{s}(z W) & \cdots & \mathrm{g}_{s}\left(z W^{r-1}\right)
\end{array}\right]=\left[\mathrm{g}_{j}\left(z W^{k}\right)\right]_{\substack{j=1,2, \ldots, s \\
k=0,1, \ldots, r-1}},
$$

where $W: \mathrm{e}^{2 \pi \mathrm{i} / r}$. In case the functions $\mathrm{g}_{j}(z), j=1,2, \ldots, s$, are Laurent polynomials, the matrix $\mathrm{G}(z)$ has Laurent polynomials entries. Besides, the relationship $\mathbb{G}(w)=\mathrm{G}\left(\mathrm{e}^{2 \pi \mathrm{i} w}\right), w \in(0,1)$, holds.

So that, we are interested in finding Laurent polynomials $\mathrm{h}_{j}(z), j=1,2 \ldots, s$, satisfying

$$
\left[\mathrm{h}_{1}(z), \mathrm{h}_{2}(z), \ldots, \mathrm{h}_{s}(z)\right] \mathrm{G}(z)=[1,0, \ldots, 0] .
$$

Thus, the trigonometric polynomials $h_{j}(w):=\mathrm{h}_{j}\left(\mathrm{e}^{2 \pi \mathrm{i} w}\right), j=1,2, \ldots, s$, satisfy (3.1), and the corresponding reconstruction vectors $c_{j, h}=\mathcal{T}_{U, a}\left(r h_{j}\right), j=1,2, \ldots, s$, 
can be expanded in $\mathcal{A}_{a}$ with just a finite number of terms. Namely,

$$
c_{j, h}=r \sum_{\text {finite }} \widehat{h}_{j}(n) U^{n} a, \quad \text { where } \mathrm{h}_{j}(z)=\sum_{\text {finite }} \widehat{h}_{j}(n) z^{n}, \quad j=1,2, \ldots, s .
$$

The following result holds:

Theorem 3.4. Assume that the sequences $\left\{\mathcal{L}_{j} a(k)\right\}_{k \in \mathbb{Z}}, j=1,2, \ldots, s$, contain only a finite number of nonzero terms. Then, there exists a vector $\mathrm{h}(z):=$ $\left[\mathrm{h}_{1}(z), \mathrm{h}_{2}(z), \ldots, \mathrm{h}_{s}(z)\right]$ whose entries are Laurent polynomials, and satisfying $\mathrm{h}(z) \mathrm{G}(z)=[1,0, \ldots, 0]$ if and only if

$$
\operatorname{rank} \mathrm{G}(z)=r \quad \text { for all } z \in \mathbb{C} \backslash\{0\} \text {. }
$$

Proof. This result is a consequence of the next lemma which proof can be found in $[34$, Theorems 5.1 and 5.6].

Lemma 3.5. Let $\mathrm{G}(z)$ be an $s \times r$ matrix whose entries are Laurent polynomials. Then, there exists an $r \times s$ matrix $\mathrm{H}(z)$ whose entries are also Laurent polynomials satisfying $\mathrm{H}(z) \mathrm{G}(z)=\mathbb{I}_{r}$ if and only if rank $\mathrm{G}(z)=r$ for all $z \in \mathbb{C} \backslash\{0\}$.

Analogously we can consider the case where the coefficients of the reconstruction vectors $c_{j, h}=r \sum_{n \in \mathbb{Z}} \widehat{h}_{j}(n) U^{n} a, j=1,2, \ldots, s$, have exponential decay, i.e. there exist $C>0$ and $q \in(0,1)$ such that $\left|\widehat{h}_{j}(n)\right| \leq C q^{|n|}, n \in \mathbb{Z}, j=1,2, \ldots, s$. Assuming that the sequences $\left\{\mathcal{L}_{j} a(k)\right\}_{k \in \mathbb{Z}}, j=1,2, \ldots, s$, have exponential decay then, we can find reconstruction vectors $c_{j, h}$ such that the sequences $\left\{\widehat{h}_{j}(n)\right\}_{n \in \mathbb{Z}}$, $j=1,2, \ldots, s$, have exponential decay if and only if $\operatorname{rank} \mathrm{G}(z)=r$ for all $z \in \mathbb{C}$ such that $|z|=1$. For the details, see $[16]$ and references therein.

\section{Time-Jitter Error: Irregular Sampling in $\mathcal{A}_{a}$}

A close look to Sec. 3 shows that all the regular sampling results have been proved without the formalism of a continuous group of unitary operators $\left\{U^{t}\right\}_{t \in \mathbb{R}}$ in $\mathcal{H}$ : we have only used the integer powers $\left\{U^{n}\right\}_{n \in \mathbb{Z}}$ which are completely determined from the unitary operator $U$. However, if we are concerned with the jitter error in a sampling formula as (3.5), the group of unitary operators becomes essential. Here, we dispose of a perturbed sequence of samples $\left\{\left(\mathcal{L}_{j} x\right)\left(r m+\epsilon_{m j}\right)\right\}_{m \in \mathbb{Z} ; j=1,2, \ldots, s}$ with errors $\epsilon_{m j} \in \mathbb{R}$, for the recovery of $x \in \mathcal{A}_{a}$. By using (2.3) and (2.2) we obtain:

$$
\begin{aligned}
\mathcal{L}_{j} x(r m) & =\left\langle F, \overline{g_{j}(w)} \mathrm{e}^{2 \pi \mathrm{i} r m w}\right\rangle_{L^{2}(0,1)} \text { and } \\
\mathcal{L}_{j} x\left(r m+\epsilon_{m j}\right) & =\left\langle F, \overline{g_{m, j}(w)} \mathrm{e}^{2 \pi \mathrm{i} r m w}\right\rangle_{L^{2}(0,1)},
\end{aligned}
$$

where the functions

$$
g_{j}(w):=\sum_{k \in \mathbb{Z}} \mathcal{L}_{j} a(k) \mathrm{e}^{2 \pi \mathrm{i} k w} \quad \text { and } \quad g_{m, j}(w):=\sum_{k \in \mathbb{Z}} \mathcal{L}_{j} a\left(k+\epsilon_{m j}\right) \mathrm{e}^{2 \pi \mathrm{i} k w},
$$

belong to $L^{2}(0,1)$. Let $\mathbb{G}(w)$ be the $s \times r$ matrix given in $(2.5)$, associated with the functions $g_{j}, j=1,2, \ldots, s$. In the case that $0<\alpha_{\mathbb{G}} \leq \beta_{\mathbb{G}}<\infty$, the sequence 
$\left\{\overline{g_{j}(w)} \mathrm{e}^{2 \pi \mathrm{i} r m w}\right\}_{m \in \mathbb{Z} ; j=1,2, \ldots, s}$ is a frame for $L^{2}(0,1)$ with optimal frame bounds $\alpha_{\mathbb{G}} / r$ and $\beta_{\mathbb{G}} / r$. Thus, as in [15], we can see the sequence $\left\{\overline{g_{m, j}(w)} \mathrm{e}^{2 \pi i r m w}\right\}_{m \in \mathbb{Z} ; j=1,2, \ldots, s}$ in $L^{2}(0,1)$ as a perturbation of the frame $\left\{\overline{g_{j}(w)} \mathrm{e}^{2 \pi \mathrm{i} r m w}\right\}_{m \in \mathbb{Z} ; j=1,2, \ldots, s}$ in $L^{2}(0,1)$. The following result on frame perturbation, which proof can be found in $[8$, p. 354] will be used later.

Lemma 4.1. Let $\left\{x_{n}\right\}_{n=1}^{\infty}$ be a frame for the Hilbert space $\mathcal{H}$ with frame bounds $A, B$, and let $\left\{y_{n}\right\}_{n=1}^{\infty}$ be a sequence in $\mathcal{H}$. If there exists a constant $R<A$ such that

$$
\sum_{n=1}^{\infty}\left|\left\langle x_{n}-y_{n}, x\right\rangle\right|^{2} \leq R\|x\|^{2} \quad \text { for each } x \in \mathcal{H}
$$

then the sequence $\left\{y_{n}\right\}_{n=1}^{\infty}$ is also a frame for $\mathcal{H}$ with bounds $A(1-\sqrt{R / A})^{2}$ and $B(1+\sqrt{R / B})^{2}$. If the sequence $\left\{x_{n}\right\}_{n=1}^{\infty}$ is a Riesz basis, then the sequence $\left\{y_{n}\right\}_{n=1}^{\infty}$ is also a Riesz basis.

\section{The time-jitter error sampling expansion}

Given an error sequence $\epsilon:=\left\{\epsilon_{m j}\right\}_{m \in \mathbb{Z} ; j=1,2, \ldots, s}$, assume that the operator

$$
\begin{aligned}
& D_{\boldsymbol{\epsilon}}: \ell^{2}(\mathbb{Z}) \rightarrow \ell_{s}^{2}(\mathbb{Z}), \\
& c=\left\{c_{l}\right\}_{l \in \mathbb{Z}} \mapsto D_{\boldsymbol{\epsilon}} c:=\left(D_{\epsilon, 1} c, \ldots, D_{\boldsymbol{\epsilon}, s} c\right),
\end{aligned}
$$

is well-defined, where, for $j=1,2, \ldots, s$,

$$
D_{\epsilon, j} c:=\left\{\sum_{k \in \mathbb{Z}}\left[\mathcal{L}_{j} a\left(r m-k+\epsilon_{m j}\right)-\mathcal{L}_{j} a(r m-k)\right] c_{k}\right\}_{m \in \mathbb{Z}} .
$$

The operator norm (it could be infinity) is defined as usual

$$
\left\|D_{\boldsymbol{\epsilon}}\right\|:=\sup _{c \in \ell^{2}(\mathbb{Z}) \backslash\{0\}} \frac{\left\|D_{\boldsymbol{\epsilon}} c\right\|_{\ell_{s}^{2}(\mathbb{Z})}}{\|c\|_{\ell^{2}(\mathbb{Z})}}
$$

where $\left\|D_{\epsilon} c\right\|_{\ell_{s}^{2}(\mathbb{Z})}^{2}:=\sum_{j=1}^{s}\left\|D_{\epsilon, j} c\right\|_{\ell^{2}(\mathbb{Z})}^{2}$ for each $c \in \ell^{2}(\mathbb{Z})$.

Theorem 4.2. Assume that for the functions $g_{j}, j=1,2, \ldots, s$, given in (2.4) we have $0<\alpha_{\mathbb{G}} \leq \beta_{\mathbb{G}}<\infty$. Let $\epsilon:=\left\{\epsilon_{m j}\right\}_{m \in \mathbb{Z} ; j=1,2, \ldots, s}$ be an error sequence satisfying the inequality $\left\|D_{\boldsymbol{\epsilon}}\right\|^{2}<\alpha_{\mathbb{G}} / r$. Then, there exists a frame $\left\{C_{j, m}^{\boldsymbol{\epsilon}}\right\}_{m \in \mathbb{Z} ; j=1,2, \ldots, s}$ for $\mathcal{A}_{a}$ such that, for any $x \in \mathcal{A}_{a}$, the sampling expansion

$$
x=\sum_{j=1}^{s} \sum_{m \in \mathbb{Z}} \mathcal{L}_{j} x\left(r m+\epsilon_{m j}\right) C_{j, m}^{\epsilon} \text { in } \mathcal{H},
$$

holds. Moreover, when $r=s$ the sequence $\left\{C_{j, m}^{\epsilon}\right\}_{m \in \mathbb{Z} ; j=1,2, \ldots, s}$ is a Riesz basis for $\mathcal{A}_{a}$, and the interpolation property $\left(\mathcal{L}_{l} C_{j, n}^{\epsilon}\right)\left(r m+\epsilon_{m j}\right)=\delta_{j, l} \delta_{n, m}$ holds. 
Proof. The sequence $\left\{\overline{g_{j}(w)} \mathrm{e}^{2 \pi \mathrm{i} r m w}\right\}_{m \in \mathbb{Z} ; j=1,2, \ldots, s}$ is a frame (a Riesz basis if $r=s$ ) for $L^{2}(0,1)$ with optimal frame (Riesz) bounds $\alpha_{\mathbb{G}} / r$ and $\beta_{\mathbb{G}} / r$. For any $F(w)=\sum_{l \in \mathbb{Z}} a_{l} \mathrm{e}^{2 \pi \mathrm{i} l w}$ in $L^{2}(0,1)$ we have

$$
\begin{aligned}
& \sum_{m \in \mathbb{Z}} \sum_{j=1}^{s}\left|\left\langle\overline{g_{m, j}(\cdot)} \mathrm{e}^{2 \pi \mathrm{i} r m}-\overline{g_{j}(\cdot)} \mathrm{e}^{2 \pi \mathrm{i} r m}, F(\cdot)\right\rangle_{L^{2}(0,1)}\right|^{2} \\
& =\sum_{m \in \mathbb{Z}} \sum_{j=1}^{s}\left|\left\langle\sum_{k \in \mathbb{Z}}\left(\overline{\mathcal{L}_{j} a\left(k+\epsilon_{m j}\right)}-\overline{\mathcal{L}_{j} a(k)}\right) \mathrm{e}^{2 \pi \mathrm{i}(r m-k)}, F(\cdot)\right\rangle_{L^{2}(0,1)}\right|^{2} \\
& =\sum_{m \in \mathbb{Z}} \sum_{j=1}^{s}\left|\left\langle\sum_{k \in \mathbb{Z}}\left(\overline{\mathcal{L}_{j} a\left(r m-k+\epsilon_{m j}\right)}-\overline{\mathcal{L}_{j} a(r m-k)}\right) \mathrm{e}^{2 \pi \mathrm{i} k \cdot}, F(\cdot)\right\rangle_{L^{2}(0,1)}\right|^{2} \\
& =\sum_{m \in \mathbb{Z}} \sum_{j=1}^{s}\left|\sum_{k \in \mathbb{Z}}\left(\overline{\mathcal{L}_{j} a\left(r m-k+\epsilon_{m j}\right)}-\overline{\mathcal{L}_{j} a(r m-k)}\right) \bar{a}_{k}\right|^{2} \\
& =\sum_{j=1}^{s}\left\|D_{\epsilon, j}\left\{a_{l}\right\}_{l \in \mathbb{Z}}\right\|_{\ell^{2}(\mathbb{Z})}^{2} \\
& \leq\left\|D_{\epsilon}\right\|^{2}\left\|\left\{a_{l}\right\}_{l \in \mathbb{Z}}\right\|_{\ell^{2}(\mathbb{Z})}^{2} \\
& =\left\|D_{\epsilon}\right\|^{2}\|F\|_{L^{2}(0,1)}^{2} \text {. }
\end{aligned}
$$

By using Lemma 4.1 we obtain that the sequence $\left\{\overline{g_{m, j}(w)} \mathrm{e}^{2 \pi \mathrm{i} r m w}\right\}_{m \in \mathbb{Z} ; j=1,2, \ldots, s}$ is a frame for $L^{2}(0,1)$ (a Riesz basis if $r=s$ ). Let $\left\{h_{j, m}^{\epsilon}\right\}_{m \in \mathbb{Z} ; j=1,2, \ldots, s}$ be its canonical dual frame. Hence, for any $F \in L^{2}(0,1)$

$$
\begin{aligned}
F & =\sum_{m \in \mathbb{Z}} \sum_{j=1}^{s}\left\langle F(\cdot), \overline{g_{m, j}(\cdot)} \mathrm{e}^{2 \pi \mathrm{i} r m \cdot}\right\rangle_{L^{2}(0,1)} h_{j, m}^{\epsilon} \\
& =\sum_{m \in \mathbb{Z}} \sum_{j=1}^{s} \mathcal{L}_{j} x\left(r m+\epsilon_{m j}\right) h_{j, m}^{\epsilon} \quad \text { in } L^{2}(0,1) .
\end{aligned}
$$

Applying the isomorphism $\mathcal{T}_{U, a}$, one gets (4.2), where $C_{j, m}^{\epsilon}:=\mathcal{T}_{U, a}\left(h_{j, m}^{\epsilon}\right)$ for $m \in \mathbb{Z}$ and $j=1,2, \ldots, s$. Since $\mathcal{T}_{U, a}$ is an isomorphism between $L^{2}(0,1)$ and $\mathcal{A}_{a}$, the sequence $\left\{C_{j, m}^{\boldsymbol{\epsilon}}\right\}_{m \in \mathbb{Z} ; j=1,2, \ldots, s}$ is a frame for $\mathcal{A}_{a}$ (a Riesz basis if $r=s$ ). The interpolatory property in the case $r=s$ follows from the uniqueness of the coefficients with respect to a Riesz basis.

Sampling formula (4.2) is useless from a practical point of view: it is impossible to determine the involved frame $\left\{C_{j, m}^{\epsilon}\right\}_{m \in \mathbb{Z} ; j=1,2, \ldots, s}$. As a consequence, in order to recover $x \in \mathcal{A}_{a}$ from the sequence of samples $\left\{\left(\mathcal{L}_{j} x\right)\left(r m+\epsilon_{m j}\right)\right\}_{m \in \mathbb{Z} ; j=1,2, \ldots, s}$ we should implement a frame algorithm in $\ell^{2}(\mathbb{Z})$ (see [15]); another possibility is given in [1]. 
In order to prove the existence of sequences $\epsilon:=\left\{\epsilon_{m j}\right\}_{m \in \mathbb{Z} ; j=1, \ldots, s}$ such that $\left\|D_{\epsilon}\right\|^{2}<\alpha_{\mathbb{G}} / r$ we need some results from the group of unitary operators theory.

\section{A brief excursion on groups of unitary operators}

Let $\left\{U^{t}\right\}_{t \in \mathbb{R}}$ denote a continuous group of unitary operators in $\mathcal{H}$. Classical Stone's theorem [26] assures us the existence of a self-adjoint operator $T$ (may be unbounded) such that $U^{t} \equiv \mathrm{e}^{\mathrm{i} t T}$. This self-adjoint operator $T$, defined on the dense domain of $\mathcal{H}$

$$
D_{T}:=\left\{x \in \mathcal{H} \text { such that } \int_{-\infty}^{\infty} w^{2} d\left\|E_{w} x\right\|^{2}<\infty\right\},
$$

admits the spectral representation $T=\int_{-\infty}^{\infty} w d E_{w}$ which means:

$$
\langle T x, y\rangle=\int_{-\infty}^{\infty} w d\left\langle E_{w} x, y\right\rangle \quad \text { for any } x \in D_{T} \text { and } y \in \mathcal{H},
$$

where $\left\{E_{w}\right\}_{w \in \mathbb{R}}$ is the corresponding resolution of the identity, i.e. a one-parameter family of projection operators $E_{w}$ in $\mathcal{H}$ such that

(i) $E_{-\infty}:=\lim _{w \rightarrow-\infty} E_{w}=O_{\mathcal{H}}, E_{\infty}:=\lim _{w \rightarrow \infty} E_{w}=I_{\mathcal{H}}$,

(ii) $E_{w^{-}}=E_{w}$ for any $-\infty<w<\infty$,

(iii) $E_{u} E_{v}=E_{w}$ where $w=\min \{u, v\}$.

Recall that $\left\|E_{w} x\right\|^{2}$ and $\left\langle E_{w} x, y\right\rangle$, as functions of $w$, have bounded variation and define, respectively, a positive and a complex Borel measure on $\mathbb{R}$.

Furthermore, for any $x \in D_{T}$ we have that $\lim _{t \rightarrow 0} \frac{U^{t} x-x}{t}=\mathrm{i} T x$ and the operator $\mathrm{i} T$ is said to be the infinitesimal generator of the group $\left\{U^{t}\right\}_{t \in \mathbb{R}}$. For each $x \in D_{T}, U^{t} x$ is a continuous differentiable function of $t$. Notice that, whenever the self-adjoint operator $T$ is bounded, $D_{T}=\mathcal{H}$ and $\mathrm{e}^{\mathrm{i} t T}$ can be defined as the usual exponential series; in any case, $U^{t} \equiv \mathrm{e}^{\mathrm{i} t T}$ means that

$$
\left\langle U^{t} x, y\right\rangle=\int_{-\infty}^{\infty} \mathrm{e}^{\mathrm{i} w t} d\left\langle E_{w} x, y\right\rangle, \quad t \in \mathbb{R}
$$

where $x \in D_{T}$ and $y \in \mathcal{H}$.

Finally, a comment on the continuity of a group of unitary operators: The group is said to be strongly continuous if, for each $x \in \mathcal{H}$ and $t_{0} \in \mathbb{R}, U^{t} x \rightarrow U^{t_{0}} x$ as $t \rightarrow t_{0}$. If $\mathcal{H}$ is a separable Hilbert space, strong continuity can be deduced from continuity and even from weak measurability, i.e. $\left\langle U^{t} x, y\right\rangle_{\mathcal{H}}$ is a Lebesgue measurable function of $t$ for any $x, y \in \mathcal{H}$. See, for instance, $[2,7,32,33]$.

On the existence of sequences $\epsilon$ such that $\left\|D_{\epsilon}\right\|^{2}<\alpha_{\mathbb{G}} / r$

Assuming that $b_{j} \in D_{T}, j=1,2, \ldots, s$, the functions $\mathcal{L}_{j} a(t), j=1,2, \ldots, s$, are continuously differentiable on $\mathbb{R}$. If, for instance, we demand in addition that, for 
each $j=1,2, \ldots, s$, there exists $\eta_{j}>0$ such that

$$
\left(\mathcal{L}_{j} a\right)^{\prime}(t)=O\left(|t|^{-\left(1+\eta_{j}\right)}\right) \quad \text { whenever }|t| \rightarrow \infty,
$$

then we can find out a finite bound for the norm $\left\|D_{\epsilon}\right\|^{2}$. Indeed, for $j=1,2, \ldots, s$ and $n, m \in \mathbb{Z}$ denote

$$
d_{m, k}^{(j)}:=\mathcal{L}_{j} a\left(r m-k+\epsilon_{m, j}\right)-\mathcal{L}_{j} a(r m-k) .
$$

Taking into account (4.1), for any sequence $c=\left\{c_{k}\right\}_{k \in \mathbb{Z}} \in \ell^{2}(\mathbb{Z})$ we have

$$
\begin{aligned}
\left\|D_{\epsilon} c\right\|_{\ell_{s}^{2}(\mathbb{Z})}^{2} & =\sum_{j=1}^{s} \sum_{m \in \mathbb{Z}}\left|\sum_{k \in \mathbb{Z}} d_{m, k}^{(j)} c_{k}\right|^{2} \\
& \leq \sum_{j=1}^{s} \sum_{m \in \mathbb{Z}} \sum_{l, k \in \mathbb{Z}}\left|d_{m, l}^{(j)} c_{l} \bar{d}_{m, k}^{(j)} \bar{c}_{k}\right| \\
& =\sum_{j=1}^{s} \sum_{l, k \in \mathbb{Z}}\left|c_{l}\right|\left|c_{k}\right| \sum_{m \in \mathbb{Z}}\left|d_{m, l}^{(j)} d_{m, k}^{(j)}\right| \\
& \leq \sum_{j=1}^{s} \sum_{l, k \in \mathbb{Z}} \frac{\left|c_{l}\right|^{2}+\left|c_{k}\right|^{2}}{2} \sum_{m \in \mathbb{Z}}\left|d_{m, l}^{(j)} d_{m, k}^{(j)}\right| \\
& =\sum_{j=1}^{s} \sum_{l \in \mathbb{Z}}\left|c_{l}\right|^{2} \sum_{k, m \in \mathbb{Z}}\left|d_{m, l}^{(j)} d_{m, k}^{(j)}\right| .
\end{aligned}
$$

Under the decay conditions (4.4), for $|\gamma| \leq 1 / 2$ we define the continuous functions,

$$
M_{\left(\mathcal{L}_{j} a\right)^{\prime}}(\gamma):=\sum_{k \in \mathbb{E}} \max _{t \in[k-\gamma, k+\gamma]}\left|\left(\mathcal{L}_{j} a\right)^{\prime}(t)\right|
$$

and

$$
N_{\left(\mathcal{L}_{j} a\right)^{\prime}}(\gamma):=\max _{k=0,1, \ldots, r-1} \sum_{m \in \mathbb{Z}} \max _{t \in[r m+k-\gamma, r m+k+\gamma]}\left|\left(\mathcal{L}_{j} a\right)^{\prime}(t)\right| .
$$

Notice that $N_{\left(\mathcal{L}_{j} a\right)^{\prime}}(\gamma) \leq M_{\left(\mathcal{L}_{j} a\right)^{\prime}}(\gamma)$ and for $r=1$ the equality holds.

Theorem 4.3. Given an error sequence $\epsilon:=\left\{\epsilon_{m j}\right\}_{m \in \mathbb{Z} ; j=1, \ldots, s}$, define the constant $\gamma_{j}:=\sup _{m \in \mathbb{Z}}\left|\epsilon_{m j}\right|$ for each $j=1,2, \ldots, s$. Then, the inequality

$$
\left\|D_{\boldsymbol{\epsilon}}\right\|^{2} \leq \sum_{j=1}^{s} \gamma_{j}^{2} N_{\left(\mathcal{L}_{j} a\right)^{\prime}}\left(\gamma_{j}\right) M_{\left(\mathcal{L}_{j} a\right)^{\prime}}\left(\gamma_{j}\right)
$$

holds and, as a consequence, condition

$$
\sum_{j=1}^{s} \gamma_{j}^{2} N_{\left(\mathcal{L}_{j} a\right)^{\prime}}\left(\gamma_{j}\right) M_{\left(\mathcal{L}_{j} a\right)^{\prime}}\left(\gamma_{j}\right)<\frac{\alpha_{\mathrm{G}}}{r}
$$

ensures the hypothesis $\left\|D_{\epsilon}\right\|^{2}<\alpha_{\mathbb{G}} / r$ in Theorem 4.2. 
Proof. For each $j=1,2, \ldots, s$, the mean value theorem gives

$$
\sup _{d \in\left[-\gamma_{j}, \gamma_{j}\right]} \sum_{n \in \mathbb{Z}}\left|\mathcal{L}_{j} a(n+d)-\mathcal{L}_{j} a(n)\right| \leq \gamma_{j} M_{\left(\mathcal{L}_{j} a\right)^{\prime}}\left(\gamma_{j}\right)
$$

and

$$
\sup _{\substack{k=0,1, \ldots, r-1 \\\left\{d_{n}\right\} \subset\left[-\gamma_{j}, \gamma_{j}\right]}} \sum_{n \in \mathbb{Z}}\left|\mathcal{L}_{j} a\left(r n+k+d_{n}\right)-\mathcal{L}_{j} a(r n+k)\right| \leq \gamma_{j} N_{\left(\mathcal{L}_{j} a\right)^{\prime}}\left(\gamma_{j}\right) .
$$

Thus, using (4.6) and (4.7), inequality (4.5) becomes

$$
\begin{aligned}
\left\|D_{\epsilon} c\right\|_{\ell_{s}^{2}(\mathbb{Z})}^{2} & \leq \sum_{j=1}^{s} \sum_{l \in \mathbb{Z}}\left|c_{l}\right|^{2} \sum_{k, m \in \mathbb{Z}}\left|d_{m, l}^{(j)} d_{m, k}^{(j)}\right| \\
& \leq \sum_{j=1}^{s} \sum_{l \in \mathbb{Z}}\left|c_{l}\right|^{2} \sum_{m \in \mathbb{Z}}\left|d_{m, l}^{(j)}\right| \gamma_{j} M_{\left(\mathcal{L}_{j} a\right)^{\prime}}\left(\gamma_{j}\right) \\
& \leq \sum_{j=1}^{s} \sum_{l \in \mathbb{Z}}\left|c_{l}\right|^{2}\left(\gamma_{j}\right)^{2} M_{\left(\mathcal{L}_{j} a\right)^{\prime}}\left(\gamma_{j}\right) N_{\left(\mathcal{L}_{j} a\right)^{\prime}}\left(\gamma_{j}\right) \\
& =\|c\|_{\ell^{2}(\mathbb{Z})}^{2} \sum_{j=1}^{s} \gamma_{j}^{2} N_{\left(\mathcal{L}_{j} a\right)^{\prime}}\left(\gamma_{j}\right) M_{\left(\mathcal{L}_{j} a\right)^{\prime}}\left(\gamma_{j}\right),
\end{aligned}
$$

which concludes the proof.

\section{The Case of Multiple Generators}

The case of $L$ generators can be analogously derived. Indeed, consider the $U$-invariant subspace generated by $\mathbf{a}:=\left\{a_{1}, a_{2}, \ldots, a_{L}\right\} \subset \mathcal{H}$, i.e.

$$
\mathcal{A}_{\mathbf{a}}:=\overline{\operatorname{span}}\left\{U^{n} a_{l}, n \in \mathbb{Z} ; l=1,2 \ldots, L\right\} .
$$

Assuming that the sequence $\left\{U^{n} a_{l}\right\}_{n \in \mathbb{Z} ; l=1,2, \ldots, L}$ is a Riesz sequence in $\mathcal{H}$, the $U$-invariant subspace $\mathcal{A}_{\mathbf{a}}$ can be expressed as

$$
\mathcal{A}_{\mathbf{a}}=\left\{\sum_{l=1}^{L} \sum_{n \in \mathbb{Z}} \alpha_{n}^{l} U^{n} a:\left\{\alpha_{n}^{l}\right\}_{n \in \mathbb{Z}} \in \ell^{2}(\mathbb{Z}) ; l=1,2 \ldots, L\right\} .
$$

The sequence $\left\{U^{n} a_{l}\right\}_{n \in \mathbb{Z} ; l=1,2, \ldots, L}$ can be thought as an $L$-dimensional stationary sequence. Its covariance matrix $\mathbf{R}_{\mathbf{a}}(k)$ is the $L \times L$ matrix

$$
\mathbf{R}_{\mathbf{a}}(k):=\left[\left\langle U^{k} a_{m}, a_{n}\right\rangle_{\mathcal{H}}\right]_{m, n=1,2, \ldots, L}, \quad k \in \mathbb{Z} .
$$

It admits the spectral representation [19]:

$$
\mathbf{R}_{\mathbf{a}}(k)=\frac{1}{2 \pi} \int_{-\pi}^{\pi} \mathrm{e}^{\mathrm{i} k \theta} d \boldsymbol{\mu}_{\mathbf{a}}(\theta), \quad k \in \mathbb{Z} .
$$

The spectral measure $\boldsymbol{\mu}_{\mathrm{a}}$ is an $L \times L$ matrix; its entries are the spectral measures associated with the cross-correlation functions $R_{m, n}(k):=\left\langle U^{k} a_{m}, a_{n}\right\rangle_{\mathcal{H}}$. It can be 
decomposed into an absolute continuous part and its singular part. Thus we can write

$$
d \boldsymbol{\mu}_{\mathbf{a}}(\theta)=\Phi_{\mathbf{a}}(\theta) d \theta+d \boldsymbol{\mu}_{\mathbf{a}}^{s}(\theta) .
$$

In case that the singular part $\boldsymbol{\mu}_{\mathrm{a}}^{s} \equiv 0$, the hermitian $L \times L$ matrix $\boldsymbol{\Phi}_{\mathbf{a}}(\theta)$ is called the spectral density of the sequence $\left\{U^{n} a_{l}\right\}_{n \in \mathbb{Z} ; l=1,2, \ldots, L}$. The following theorem holds.

Theorem 5.1. Let $\left\{U^{n} a_{l}\right\}_{n \in \mathbb{Z} ; l=1,2, \ldots, L}$ be a sequence obtained from a unitary operator in a separable Hilbert space $\mathcal{H}$ with spectral measure $d \boldsymbol{\mu}_{\mathbf{a}}(\theta)=\Phi_{\mathbf{a}}(\theta) d \theta+$ $d \boldsymbol{\mu}_{\mathbf{a}}^{s}(\theta)$, and let $\mathcal{A}_{\mathbf{a}}$ be the closed subspace spanned by $\left\{U^{n} a_{l}\right\}_{n \in \mathbb{Z} ; l=1,2, \ldots, L}$. Then the sequence $\left\{U^{n} a_{l}\right\}_{n \in \mathbb{Z} ; l=1,2, \ldots, L}$ is a Riesz basis for $\mathcal{A}_{\mathbf{a}}$ if and only if the singular part $\boldsymbol{\mu}_{\mathbf{a}}^{s} \equiv 0$ and

$$
0<\underset{\theta \in(-\pi, \pi)}{\operatorname{essinf}} \lambda_{\min }\left[\Phi_{\mathbf{a}}(\theta)\right] \leq \operatorname{essip}_{\theta \in(-\pi, \pi)} \lambda_{\max }\left[\Phi_{\mathbf{a}}(\theta)\right]<\infty .
$$

Proof. For a fixed $\ell_{L}^{2}$-sequence $c:=\left\{c_{n}^{l}\right\}_{n \in \mathbb{Z} ; l=1,2, \ldots, L}$ we have

$$
\begin{aligned}
\left\|\sum_{l=1}^{L} \sum_{k \in \mathbb{Z}} c_{k}^{l} U^{k} a_{l}\right\|^{2} & =\sum_{i, j=1}^{L} \sum_{m \in \mathbb{Z}} \sum_{n \in \mathbb{Z}} c_{m}^{i} \bar{c}_{m}^{j}\left\langle U^{m} a_{i}, U^{n} a_{j}\right\rangle \\
& =\sum_{i, j=1}^{L} \sum_{m \in \mathbb{Z}} \sum_{n \in \mathbb{Z}} c_{m}^{i} \bar{c}_{n}^{j} \frac{1}{2 \pi} \int_{-\pi}^{\pi} \mathrm{e}^{\mathrm{i} m \theta} \mathrm{e}^{-\mathrm{i} n \theta} d \mu_{a_{i}, a_{j}}(\theta) \\
& =\frac{1}{2 \pi} \int_{-\pi}^{\pi} \sum_{m \in \mathbb{Z}} \sum_{n \in \mathbb{Z}}\left(\mathbf{c}_{m} \mathrm{e}^{\mathrm{i} m \theta}\right)^{\top} d \boldsymbol{\mu}_{\mathbf{a}}(\theta) \overline{\mathbf{c}}_{n} \mathrm{e}^{-\mathrm{i} n \theta},
\end{aligned}
$$

where $\mathbf{c}_{k}=\left(c_{k}^{1}, c_{k}^{2}, \ldots, c_{k}^{L}\right)^{\top}$ for every $k \in \mathbb{Z}$.

First, we show that if the measure $\mu_{\mathbf{a}}$ is not absolutely continuous with respect to Lebesgue measure $\lambda$ then $\left\{U^{n} a_{l}\right\}_{n \in \mathbb{Z} ; l=1,2, \ldots, L}$ is not a Riesz basis for $\mathcal{A}_{\mathbf{a}}$. Indeed, if the spectral measure $\boldsymbol{\mu}_{\mathrm{a}}$ is not absolutely continuous with respect to Lebesgue measure then there exists $i \in\{1,2, \ldots, L\}$ such that the positive spectral measure $\mu_{a_{i}, a_{i}}$ is not absolutely continuous with respect to Lebesgue measure; this comes from the fact that, if any spectral measure in the diagonal $\mu_{a_{j}, a_{j}}$ is absolutely continuous with respect to Lebesgue measure, the same occurs for each measure $\mu_{a_{j}, a_{k}}$ with $k \neq j$ (see $\left[7\right.$, p. 137]). Then, $\mu_{a_{i}, a_{i}}(B)>0$ for a (Lebesgue) measurable set $B \subset(-\pi, \pi)$ of Lebesgue measure zero. Bearing in mind that every measurable set is included in a Borel set, actually an intersection of a countable collection of open sets, having the same Lebesgue measure (see [25, p. 63]), we take $B$ to be a Borel set. Moreover, since every finite Borel measure on $(-\pi, \pi)$ is inner regular (see $[25$, p. 340]) we may also assume that $B$ is a compact set. For any $\varepsilon>0$ there exists a sequence of disjoint open intervals $I_{j} \subset(-\pi, \pi)$ such that

$$
B \subset \bigcup_{j=1}^{\infty} I_{j} \quad \text { and } \quad \sum_{j=1}^{\infty} \lambda\left(I_{j}\right) \leq \lambda(B)+\varepsilon=\varepsilon,
$$


(see [25, pp. 58 and 42$]$ ). Since $B$ is compact we may take the sequence to be finite. Hence, for every $N \in \mathbb{N}$ there exist open disjoint intervals $I_{1}^{N}, I_{2}^{N}, \ldots, I_{j_{N}}^{N}$ in $(-\pi, \pi)$ such that

$$
B \subset \bigcup_{j=1}^{j_{N}} I_{j}^{N} \text { and } \quad \sum_{j=1}^{j_{N}} \lambda\left(I_{j}^{N}\right) \leq \frac{1}{3^{N}}
$$

Besides, $\sum_{j=1}^{j_{N}} \mu_{a_{i}, a_{i}}\left(I_{j}^{N}\right) \geq \mu_{a_{i}, a_{i}}(B)$. Consider the function $g_{N}:(-\pi, \pi) \rightarrow \mathbb{R}$, where $g_{N}=2^{N / 2} \chi_{\bigcup_{j=1}^{j_{N}} I_{j}^{N}}$, that satisfies

$$
\left\|g_{N}\right\|_{2}^{2}=2^{N} \sum_{j=1}^{j_{N}} \lambda\left(I_{j}^{N}\right) \leq \frac{2^{N}}{3^{N}}<1 .
$$

We modify and extend each $g_{N}$ to obtain a $2 \pi$-periodic function $f_{N}: \mathbb{R} \rightarrow \mathbb{R}$ such that $f_{N}$ and its derivative are continuous on $\mathbb{R},\left\|f_{N}\right\|_{2}^{2} \leq 1$ and $f_{N}(\theta)=g_{N}(\theta)$ for every $\theta \in \bigcup_{j=1}^{j_{N}} I_{j}^{N}$. Let $\sum_{k} c_{k}^{N} \mathrm{e}^{\mathrm{i} k \theta}$ be the Fourier series of $f_{N}$. First, by using Parseval's identity we have

$$
\left\|c_{k}^{N}\right\|_{2}^{2}=\frac{1}{2 \pi}\left\|f_{N}\right\|_{2}^{2} \leq \frac{1}{2 \pi} \quad \text { for every } N \in \mathbb{N},
$$

so that $\left\{c^{N}\right\}_{N=1}^{\infty}$ is a bounded sequence in $\ell^{2}(\mathbb{Z})$. Besides, the regularity of each $f_{N}$ ensures that each Fourier series converges uniformly to $f_{N}$. Therefore, each series $\sum_{k} c_{k}^{N} \mathrm{e}^{\mathrm{i} k \theta}$ converges to $f_{N}$ in $L_{\mu_{a_{i}, a_{i}}(-\pi, \pi)}^{2}$ and consequently,

$$
\begin{aligned}
\left\|\sum_{k} c_{k}^{N} \mathrm{e}^{\mathrm{i} k \theta}\right\|_{L_{\mu_{a_{i}, a_{i}}^{2}}^{2}(-\pi, \pi)}^{2} & =\int_{-\pi}^{\pi}\left|f_{N}\right|^{2} d \mu_{a_{i}, a_{i}} \geq \int_{-\pi}^{\pi}\left|g_{N}\right|^{2} d \mu_{a_{i}, a_{i}} \\
& =2^{N} \sum_{j=1}^{j_{N}} \mu_{a_{i}, a_{i}}\left(I_{j}^{N}\right) \geq 2^{N} \mu_{a_{i}, a_{i}}(B) .
\end{aligned}
$$

For every $c^{N} \in \ell^{2}(\mathbb{Z})$ we consider the $\ell_{L}^{2}$-sequence $\left\{c_{n}^{N l}\right\}_{n \in \mathbb{Z} ; l=1,2, \ldots, L}$ given by $c_{n}^{N i}=c_{n}^{N}$ and $c_{n}^{N l}=0$ if $l \neq i$. Substituting each $\left\{c_{n}^{N l}\right\}_{n \in \mathbb{Z} ; l=1,2, \ldots, L}$ in (5.2) we have that

$$
\left\|\sum_{l=1}^{L} \sum_{k \in \mathbb{Z}} c_{k}^{N l} U^{k} a_{l}\right\|^{2}=\frac{1}{2 \pi} \int_{-\pi}^{\pi}\left|\sum_{k \in \mathbb{Z}} c_{k}^{N} \mathrm{e}^{\mathrm{i} k \theta}\right|^{2} d \mu_{a_{i}, a_{i}}(\theta)
$$

tends to infinity with $N$, so $\left\{U^{n} a_{l}\right\}_{n \in \mathbb{Z} ; l=1,2, \ldots, L}$ cannot be a Bessel sequence, therefore, not a Riesz basis.

For the remainder of the proof we assume that the singular part $\mu_{\mathrm{a}}^{s} \equiv 0$ and that $d \boldsymbol{\mu}_{\mathbf{a}}(\theta)=\boldsymbol{\Phi}_{\mathbf{a}}(\theta) d \theta$. Then (5.2) yields that

$$
\left\|\sum_{l=1}^{L} \sum_{k \in \mathbb{Z}} c_{k}^{l} U^{k} a_{l}\right\|^{2}=\frac{1}{2 \pi} \int_{-\pi}^{\pi}\left(\sum_{m \in \mathbb{Z}} \mathbf{c}_{m} \mathrm{e}^{\mathrm{i} m \theta}\right)^{\top} \boldsymbol{\Phi}_{\mathbf{a}}(\theta) \overline{\sum_{n \in \mathbb{Z}} \mathbf{c}_{n} \mathrm{e}^{\mathrm{i} n \theta}} d \theta .
$$


We have to show that $\left\{U^{n} a_{l}\right\}_{n \in \mathbb{Z} ; l=1,2, \ldots, L}$ is a Riesz basis for $\mathcal{A}_{\mathbf{a}}$ if and only if $(5.1)$ holds. Rayleigh-Ritz theorem (see [17, p. 176]) provides the inequalities

$$
\begin{aligned}
\lambda_{\min }\left[\boldsymbol{\Phi}_{\mathbf{a}}(\theta)\right]\left|\sum_{k \in \mathbb{Z}} \mathrm{c}_{k} \mathrm{e}^{\mathrm{i} k \theta}\right|^{2} & \leq\left(\sum_{m \in \mathbb{Z}} \mathbf{c}_{m} \mathrm{e}^{\mathrm{i} m \theta}\right)^{\top} \boldsymbol{\Phi}_{\mathbf{a}}(\theta) \overline{\sum_{n \in \mathbb{Z}} \mathbf{c}_{n} \mathrm{e}^{\mathrm{i} n \theta}} \\
& \leq \lambda_{\max }\left[\boldsymbol{\Phi}_{\mathbf{a}}(\theta)\right]\left|\sum_{k \in \mathbb{Z}} \mathbf{c}_{k} \mathrm{e}^{\mathrm{i} k \theta}\right|^{2},
\end{aligned}
$$

and taking into account (5.3) we have

$$
\begin{aligned}
\frac{1}{2 \pi} \int_{-\pi}^{\pi} \lambda_{\min }\left[\mathbf{\Phi}_{\mathbf{a}}(\theta)\right]\left|\sum_{k \in \mathbb{Z}} \mathbf{c}_{k} \mathrm{e}^{\mathrm{i} k \theta}\right|^{2} d \theta & \leq\left\|\sum_{l=1}^{L} \sum_{k \in \mathbb{Z}} c_{k}^{l} U^{k} a_{l}\right\|^{2} \\
& \leq \frac{1}{2 \pi} \int_{-\pi}^{\pi} \lambda_{\max }\left[\Phi_{\mathbf{a}}(\theta)\right]\left|\sum_{k \in \mathbb{Z}} \mathrm{c}_{k} \mathrm{e}^{\mathrm{i} k \theta}\right|^{2} d \theta
\end{aligned}
$$

so that

$$
\begin{aligned}
\underset{\theta \in(-\pi, \pi)}{\operatorname{essinf}} \lambda_{\min }\left[\Phi_{\mathbf{a}}(\theta)\right] \sum_{l=1}^{L} \sum_{k \in \mathbb{Z}}\left|c_{k}^{l}\right|^{2} & \leq\left\|\sum_{l=1}^{L} \sum_{k \in \mathbb{Z}} c_{k}^{l} U^{k} a_{l}\right\|^{2} \\
& \leq \operatorname{ess~sup}_{\theta \in(-\pi, \pi)} \lambda_{\max }\left[\Phi_{\mathbf{a}}(\theta)\right] \sum_{l=1}^{L} \sum_{k \in \mathbb{Z}}\left|c_{k}^{l}\right|^{2} .
\end{aligned}
$$

Therefore, (5.1) implies that $\left\{U^{n} a_{l}\right\}_{n \in \mathbb{Z} ; l=1,2, \ldots, L}$ is a Riesz basis for $\mathcal{A}_{\mathbf{a}}$.

Conversely, if $\left\{U^{n} a_{l}\right\}_{n \in \mathbb{Z} ; l=1,2, \ldots, L}$ is a Riesz basis for $\mathcal{A}_{\mathbf{a}}$ then there exist constants $0<A \leq B<\infty$ such that

$$
A \sum_{l=1}^{L} \sum_{k \in \mathbb{Z}}\left|c_{k}^{l}\right|^{2} \leq\left\|\sum_{l=1}^{L} \sum_{k \in \mathbb{Z}} c_{k}^{l} U^{k} a_{l}\right\|^{2} \leq B \sum_{l=1}^{L} \sum_{k \in \mathbb{Z}}\left|c_{k}^{l}\right|^{2}
$$

for every $\ell_{L}^{2}$-sequence $c:=\left\{c_{n}^{l}\right\}_{n \in \mathbb{Z} ; l=1,2, \ldots, L}$. Let us prove that

$$
\left.A \leq \underset{\theta \in(-\pi, \pi)}{\operatorname{essinf}} \lambda_{\min }\left[\boldsymbol{\Phi}_{\mathbf{a}}(\theta)\right] \leq \underset{\theta \in(-\pi, \pi)}{\operatorname{ess} \sup _{\max }} \lambda_{\mathbf{a}}(\theta)\right] \leq B
$$

Proceeding by contradiction, if (5.5) would not hold, then

$$
A \leq \lambda_{\min }\left[\Phi_{\mathrm{a}}(\theta)\right] \leq \lambda_{\max }\left[\boldsymbol{\Phi}_{\mathrm{a}}(\theta)\right] \leq B
$$

does not hold on a subset of $(-\pi, \pi)$ with positive Lebesgue measure. In case the set $\Gamma_{B}:=\left\{\theta \in(-\pi, \pi): \lambda_{\max }\left[\Phi_{\mathbf{a}}(\theta)\right]>B\right\}$ has positive Lebesgue measure we introduce the Fourier expansion of the function $F \in L_{L}^{2}(-\pi, \pi)\left(L_{L}^{2}(-\pi, \pi)\right.$ denotes the usual product Hilbert space $L^{2}(-\pi, \pi) \times \cdots \times L^{2}(-\pi, \pi)(L$ times $\left.)\right)$ in $(5.3)$, 
where $F(\theta)=\mathbf{X}(\theta) \chi_{\Gamma_{B}}(\theta)$ and $\mathbf{X}(\theta)$ is an eigenvector of norm 1 associated with the biggest eigenvalue of $\boldsymbol{\Phi}_{\mathbf{a}}(\theta)$. We get

$$
\left\|\sum_{l=1}^{L} \sum_{k \in \mathbb{Z}} c_{k}^{l} U^{k} a_{l}\right\|^{2}=\frac{1}{2 \pi} \int_{\Gamma_{B}} \lambda_{\max }\left[\Phi_{\mathbf{a}}(\theta)\right] d \theta>\frac{1}{2 \pi} \int_{\Gamma_{B}} B d \theta
$$

which contradicts the right inequality in (5.4) for such a Fourier expansion. Whenever Lebesgue measure of the set $\Gamma_{B}$ is zero then we proceed in a similar way with the set of positive Lebesgue measure $\Gamma_{A}:=\left\{\theta \in(-\pi, \pi): \lambda_{\min }\left[\boldsymbol{\Phi}_{\mathbf{a}}(\theta)\right]<A\right\}$.

The above proof is similar to that of [24, Lemma 2], except we do not exclude the case in which the singular measure is atomless. Another characterization for being $\left\{U^{n} a_{l}\right\}_{n \in \mathbb{Z} ; l=1,2, \ldots, L}$ a Riesz basis for $\mathcal{A}_{\mathbf{a}}$ can be found in [3].

The resulting regular sampling formulas

As in the one-generator case, the space $\mathcal{A}_{\mathbf{a}}$ is the image of the usual product Hilbert space $L_{L}^{2}(0,1)$ by means of the isomorphism $\mathcal{T}_{U, \mathbf{a}}: L_{L}^{2}(0,1) \longrightarrow \mathcal{A}_{\mathbf{a}}$, which maps the orthonormal basis $\left\{\mathrm{e}^{-2 \pi i n w} \mathbf{e}_{l}\right\}_{n \in \mathbb{Z} ; l=1,2, \ldots, L}$ for $L_{L}^{2}(0,1)$ (here, $\left\{\mathbf{e}_{l}\right\}_{l=1}^{L}$ denotes the canonical basis for $\mathbb{C}^{L}$ ) onto the Riesz basis $\left\{U^{n} a_{l}\right\}_{n \in \mathbb{Z} ; l=1,2, \ldots, L}$ for $\mathcal{A}_{\mathbf{a}}$, i.e.

$$
\mathcal{T}_{U, \mathbf{a}} \mathbf{F}:=\sum_{l=1}^{L} \sum_{n \in \mathbb{Z}}\left\langle F_{l}, \mathrm{e}^{2 \pi \mathrm{i} n}\right\rangle_{L^{2}(0,1)} U^{n} a_{l}=\sum_{l=1}^{L} \sum_{n \in \mathbb{Z}} \alpha_{n}^{l} U^{n} a_{l},
$$

where $\mathbf{F}=\left(F_{1}, F_{2}, \ldots, F_{L}\right)^{\top} \in L_{L}^{2}(0,1)$.

Here, for $\mathbf{F} \in L_{L}^{2}(0,1)$ and $N \in \mathbb{Z}$ the $U$-shift property reads:

$$
\mathcal{T}_{U, \mathbf{a}}\left(\mathbf{F} \mathrm{e}^{2 \pi \mathrm{i} N w}\right)=U^{N}\left(\mathcal{T}_{U, \mathbf{a}} \mathbf{F}\right)
$$

Concerning the representation of an $U$-system $\mathcal{L}_{b}$, for $x \in \mathcal{A}_{\mathbf{a}}$ we have

$$
\begin{aligned}
\mathcal{L}_{b} x(t) & =\left\langle x, U^{t} b\right\rangle_{\mathcal{H}}=\sum_{l=1}^{L} \sum_{n \in \mathbb{Z}} \alpha_{n}^{l}{\overline{\left\langle U^{t} b, U^{n} a_{l}\right\rangle_{\mathcal{H}}}} \\
& =\sum_{l=1}^{L}\left\langle F_{l}, \sum_{n \in \mathbb{Z}}\left\langle U^{t} b, U^{n} a_{l}\right\rangle_{\mathcal{H}} \mathrm{e}^{2 \pi \mathrm{in} w}\right\rangle_{L^{2}(0,1)}=\left\langle\mathbf{F}, \mathbf{K}_{t}\right\rangle_{L_{L}^{2}(0,1)},
\end{aligned}
$$

where $\mathcal{T}_{U, a} \mathbf{F}=x, \mathbf{F}=\left(F_{1}, F_{2}, \ldots, F_{L}\right)^{\top} \in L_{L}^{2}(0,1)$, and the function

$$
\begin{aligned}
\mathbf{K}_{t}(w):= & \left(\sum_{n \in \mathbb{Z}} \overline{\mathcal{L}_{b} a_{1}(t-n)} \mathrm{e}^{2 \pi \mathrm{i} n w}, \sum_{n \in \mathbb{Z}} \overline{\mathcal{L}_{b} a_{2}(t-n)} \mathrm{e}^{2 \pi \mathrm{i} n w}, \ldots,\right. \\
& \left.\sum_{n \in \mathbb{Z}} \overline{\mathcal{L}_{b} a_{L}(t-n)} \mathrm{e}^{2 \pi \mathrm{i} n w}\right)^{\top}
\end{aligned}
$$


belongs to $L_{L}^{2}(0,1)$. In particular, given $s U$-systems $\mathcal{L}_{j}:=\mathcal{L}_{b_{j}}$ associated with $b_{j}$ in $\mathcal{H}, j=1,2, \ldots, s$, we get the expression for the samples $\left\{\mathcal{L}_{j} x(r m)\right\}_{m \in \mathbb{Z} ; j=1,2, \ldots, s}$ :

$$
\mathcal{L}_{j} x(r m)=\left\langle\mathbf{F}, \overline{\mathbf{g}_{j}(w)} \mathrm{e}^{2 \pi i r m w}\right\rangle_{L_{L}^{2}(0,1)} \quad \text { for } m \in \mathbb{Z} \quad \text { and } \quad j=1,2, \ldots, s,
$$

where $\mathcal{T}_{U, a} \mathbf{F}=x$ and for $j=1,2, \ldots, s$

$$
\mathbf{g}_{j}(w):=\left(\sum_{k \in \mathbb{Z}} \mathcal{L}_{j} a_{1}(k) \mathrm{e}^{2 \pi \mathrm{i} k w}, \sum_{k \in \mathbb{Z}} \mathcal{L}_{j} a_{2}(k) \mathrm{e}^{2 \pi \mathrm{i} k w}, \ldots, \sum_{k \in \mathbb{Z}} \mathcal{L}_{j} a_{L}(k) \mathrm{e}^{2 \pi \mathrm{i} k w}\right)^{\top}
$$

belongs to $L_{L}^{2}(0,1)$. As in the one-generator case we must study the sequence $\left\{\overline{\mathbf{g}_{j}(w)} \mathrm{e}^{2 \pi \mathrm{i} r m w}\right\}_{m \in \mathbb{Z} ; j=1,2, \ldots, s}$ in $L_{L}^{2}(0,1)$. Consider the $s \times r L$ matrix of functions in $L^{2}(0,1)$

$$
\begin{aligned}
& \mathbb{G}(w):=\left[\begin{array}{cccc}
\mathbf{g}_{1}^{\top}(w) & \mathbf{g}_{1}^{\top}\left(w+\frac{1}{r}\right) & \cdots & \mathbf{g}_{1}^{\top}\left(w+\frac{r-1}{r}\right) \\
\mathbf{g}_{2}^{\top}(w) & \mathbf{g}_{2}^{\top}\left(w+\frac{1}{r}\right) & \cdots & \mathbf{g}_{2}^{\top}\left(w+\frac{r-1}{r}\right) \\
\vdots & \vdots & & \vdots \\
\mathbf{g}_{s}^{\top}(w) & \mathbf{g}_{s}^{\top}\left(w+\frac{1}{r}\right) & \cdots & \mathbf{g}_{s}^{\top}\left(w+\frac{r-1}{r}\right)
\end{array}\right] \\
& =\left[\mathbf{g}_{j}^{\top}\left(w+\frac{k-1}{r}\right)\right]_{\substack{j=1,2, \ldots, s \\
k=1,2, \ldots, r}}
\end{aligned}
$$

and its related constants

$$
\alpha_{\mathbb{G}}:=\underset{w \in(0,1 / r)}{\operatorname{essinf}} \lambda_{\min }\left[\mathbb{G}^{*}(w) \mathbb{G}(w)\right], \quad \beta_{\mathbb{G}}:=\operatorname{ess}_{w \in(0,1 / r)} \lambda_{\max }\left[\mathbb{G}^{*}(w) \mathbb{G}(w)\right] .
$$

In [13, Lemma 2], one can find the proof of the following lemma.

Lemma 5.2. Let $\mathbf{g}_{j}$ be in $L_{L}^{2}(0,1)$ for $j=1,2, \ldots, s$ and let $\mathbb{G}(w)$ be its associated matrix given in (5.9). Then, the following results hold:

(a) The sequence $\left\{\overline{\mathbf{g}_{j}(w)} \mathrm{e}^{2 \pi \mathrm{i} r n w}\right\}_{n \in \mathbb{Z}, j=1,2, \ldots, s}$ is a complete system for $L_{L}^{2}(0,1)$ if and only if the rank of the matrix $\mathbb{G}(w)$ is $r L$ a.e. in $(0,1 / r)$.

(b) The sequence $\left\{\overline{\mathbf{g}_{j}(w)} \mathrm{e}^{2 \pi \mathrm{i} r n w}\right\}_{n \in \mathbb{Z}, j=1,2, \ldots, s}$ is a Bessel sequence for $L_{L}^{2}(0,1)$ if and only if $\mathbf{g}_{j} \in L_{L}^{\infty}(0,1)$ (or equivalently $\beta_{\mathbb{G}}<\infty$ ). In this case, the optimal Bessel bound is $\beta_{\mathbb{G}} / r$.

(c) The sequence $\left\{\overline{\mathbf{g}_{j}(w)} \mathrm{e}^{2 \pi \mathrm{irnw}}\right\}_{n \in \mathbb{Z}, j=1,2, \ldots, s}$ is a frame for $L_{L}^{2}(0,1)$ if and only if $0<\alpha_{\mathbb{G}} \leq \beta_{\mathbb{G}}<\infty$. In this case, the optimal frame bounds are $\alpha_{\mathbb{G}} / r$ and $\beta_{\mathbb{G}} / r$.

(d) The sequence $\left\{\overline{\mathbf{g}_{j}(w)} \mathrm{e}^{2 \pi \mathrm{i} r n w}\right\}_{n \in \mathbb{Z}, j=1,2, \ldots, s}$ is a Riesz basis for $L_{L}^{2}(0,1)$ if and only if is a frame and $s=r L$.

In case that the sequence $\left\{\overline{\mathbf{g}_{j}(w)} \mathrm{e}^{2 \pi \mathrm{i} r n w}\right\}_{n \in \mathbb{Z} ; j=1,2, \ldots, s}$ is a frame for $L_{L}^{2}(0,1)$ (here, necessarily $s \geq r L$ ), a dual frame is given by $\left\{r \mathbf{h}_{j}(w) \mathrm{e}^{2 \pi i r n w}\right\}_{n \in \mathbb{Z} ; j=1,2, \ldots, s}$, 
where the functions $\mathbf{h}_{j}, j=1,2, \ldots, s$, form an $L \times s$ matrix $\mathbf{h}(w):=\left[\mathbf{h}_{1}(w)\right.$, $\left.\mathbf{h}_{2}(w), \ldots, \mathbf{h}_{s}(w)\right]$ with entries in $L^{\infty}(0,1)$, and satisfying

$$
\left[\mathbf{h}_{1}(w), \mathbf{h}_{2}(w), \ldots, \mathbf{h}_{s}(w)\right] \mathbb{G}(w)=\left[\mathbb{I}_{L}, \mathbb{O}_{L \times(r-1) L}\right] \quad \text { a.e. in }(0,1)
$$

(see [13] for the details). That is, the matrix $\mathbf{h}(w)$ is formed with the first $L$ rows of a left-inverse of the matrix $\mathbb{G}(w)$ having essentially bounded entries in $(0,1)$. In other words, all the dual frames of $\left\{\overline{\mathbf{g}_{j}\left(\mathrm{e}^{2 \pi \mathrm{irnw}}\right)}\right\}_{n \in \mathbb{Z} ; j=1,2, \ldots, s}$ with the above property are obtained by taking the first $L$ rows of the $r L \times s$ matrices given by

$$
\mathbb{H}_{\mathbb{K}}(w):=\mathbb{G}^{\dagger}(w)+\mathbb{K}(w)\left[\mathbb{I}_{s}-\mathbb{G}(w) \mathbb{G}^{\dagger}(w)\right],
$$

where $\mathbb{K}(w)$ denotes any $r L \times s$ matrix with entries in $L^{\infty}(0,1)$.

Thus, any $\mathbf{F} \in L_{L}^{2}(0,1)$ can be expanded as

$$
\mathbf{F}=\sum_{j=1}^{s} \sum_{n \in \mathbb{Z}}\left\langle\mathbf{F}, \overline{\mathbf{g}_{j}(w)} \mathrm{e}^{2 \pi \mathrm{i} r n w}\right\rangle_{L_{L}^{2}(0,1)} r \mathbf{h}_{j}(w) \mathrm{e}^{2 \pi \mathrm{i} r n w} \quad \text { in } L_{L}^{2}(0,1) .
$$

Applying the isomorphism $\mathcal{T}_{U, a}$ and taken into account (5.8), for each $x=\mathcal{T}_{U, a} \mathbf{F} \in$ $\mathcal{A}_{\mathbf{a}}$ we get the sampling expansion

$$
x=\sum_{j=1}^{s} \sum_{n \in \mathbb{Z}} \mathcal{L}_{j} x(r n) U^{r n}\left[\mathcal{T}_{U, a}\left(r \mathbf{h}_{j}\right)\right]=\sum_{j=1}^{s} \sum_{n \in \mathbb{Z}} \mathcal{L}_{j} x(r n) U^{r n} c_{j, \mathbf{h}} \quad \text { in } \mathcal{H},
$$

where the sampling elements $c_{j, \mathbf{h}}=\mathcal{T}_{U, a}\left(r \mathbf{h}_{j}\right) \in \mathcal{A}_{\mathbf{a}}, j=1,2, \ldots, s$, and the sequence $\left\{U^{r n} c_{j, \mathbf{h}}\right\}_{n \in \mathbb{Z} ; j=1,2, \ldots, s}$ is a frame for $\mathcal{A}_{\mathbf{a}}$. Proceeding as in Sec. 3 , it is straightforward to state and prove the corresponding results.

\section{The time-jitter error sampling formulas}

Under appropriate slight changes, the time-jitter error results in Sec. 4 still remain valid for the case of multiple generators. Namely, given an error sequence $\epsilon:=$ $\left\{\epsilon_{m j}\right\}_{m \in \mathbb{Z} ; j=1,2, \ldots, s}$, assume that the operator

$$
\begin{aligned}
D_{\boldsymbol{\epsilon}}: \ell_{L}^{2}(\mathbb{Z}) & \rightarrow \ell_{s}^{2}(\mathbb{Z}), \\
\mathbf{c} & \mapsto D_{\boldsymbol{\epsilon}} \mathbf{c}:=\left(D_{\boldsymbol{\epsilon}, 1} \mathbf{c}, \ldots, D_{\boldsymbol{\epsilon}, s} \mathbf{c}\right),
\end{aligned}
$$

is well-defined, where $\mathrm{c}:=\left(\left\{c_{k}^{1}\right\}_{k \in \mathbb{Z}},\left\{c_{k}^{2}\right\}_{k \in \mathbb{Z}}, \ldots,\left\{c_{k}^{L}\right\}_{k \in \mathbb{Z}}\right) \in \ell_{L}^{2}(\mathbb{Z})$ and, for $j=$ $1,2, \ldots, s$,

$$
D_{\epsilon, j} \mathrm{c}:=\left\{\sum_{l=1}^{L} \sum_{k \in \mathbb{Z}}\left[\mathcal{L}_{j} a_{l}\left(r m-k+\epsilon_{m j}\right)-\mathcal{L}_{j} a_{l}(r m-k)\right] c_{k}^{l}\right\}_{m \in \mathbb{Z}} .
$$

The operator norm (it could be infinity) is defined as usual

$$
\left\|D_{\epsilon}\right\|:=\sup _{\mathbf{c} \in \ell_{L}^{2}(\mathbb{Z}) \backslash\{0\}} \frac{\left\|D_{\epsilon} \mathbf{c}\right\|_{\ell_{s}^{2}(\mathbb{Z})}}{\|\mathbf{c}\|_{\ell_{L}^{2}(\mathbb{Z})}}
$$

where $\left\|D_{\epsilon} \mathbf{c}\right\|_{\ell_{s}^{2}(\mathbb{Z})}^{2}:=\sum_{j=1}^{s}\left\|D_{\epsilon, j} \mathbf{c}\right\|_{\ell^{2}(\mathbb{Z})}^{2}$ and $\|\mathbf{c}\|_{\ell_{L}^{2}(\mathbb{Z})}^{2}=\sum_{l=1}^{L} \sum_{k \in \mathbb{Z}}\left|c_{k}^{l}\right|^{2}$ for each $\mathrm{c} \in \ell_{L}^{2}(\mathbb{Z})$. Assume that the matrix $\mathbb{G}$ in $(5.9)$ satisfies $0<\alpha_{\mathbb{G}} \leq \beta_{\mathbb{G}}<\infty$, and 
let $\epsilon:=\left\{\epsilon_{m j}\right\}_{m \in \mathbb{Z} ; j=1,2, \ldots, s}$ be an error sequence satisfying the inequality $\left\|D_{\epsilon}\right\|^{2}<$ $\alpha_{\mathbb{G}} / r$. Then, proceeding as in Sec. 4, there exists a frame $\left\{C_{j, m}^{\epsilon}\right\}_{m \in \mathbb{Z} ; j=1,2, \ldots, s}$ for $\mathcal{A}_{\mathbf{a}}$ such that, for any $x \in \mathcal{A}_{\mathbf{a}}$ a sampling formula as in (4.2) holds.

Now assume that $b_{j} \in D_{T}, j=1,2, \ldots, s$; thus the functions $\mathcal{L}_{b_{j}} a_{l}(t) \equiv \mathcal{L}_{j} a_{l}(t)$, $j=1,2, \ldots, s$ and $l=1,2, \ldots, L$, are continuously differentiable on $\mathbb{R}$. Again, as in Sec. 4, under the decay condition (4.4) for each $\left(\mathcal{L}_{j} a_{l}\right)^{\prime}(t), j=1,2, \ldots, s$ and $l=1,2, \ldots, L$, one can easily prove that there exists $\delta>0$ such that $\gamma_{j}:=$ $\sup _{m \in \mathbb{Z}}\left|\epsilon_{m j}\right|<\delta$ for each $j=1,2, \ldots, s$, implies that $\left\|D_{\epsilon}\right\|^{2}<\alpha_{\mathbb{G}} / r$ for the error sequence $\boldsymbol{\epsilon}:=\left\{\epsilon_{m j}\right\}_{m \in \mathbb{Z} ; j=1,2, \ldots, s}$.

\section{Acknowledgments}

The authors wish to thank the referees for their valuable and constructive comments. This work has been supported by the grant MTM2009-08345 from the Spanish Ministerio de Ciencia e Innovación (MICINN).

\section{References}

[1] B. Adcock and A. C. Hansen, Stable reconstructions in Hilbert spaces and the resolution of the Gibbs phenomenon, Appl. Comput. Harmon. Anal. 32 (2012) 357-388.

[2] N. I. Akhiezer and I. M. Glazman, Theory of Linear Operators in Hilbert Space (Dover Publications, New York, 1993).

[3] A. Aldroubi, Oblique projections in atomic spaces, Proc. Amer. Math. Soc. 124(7) (1996) 2051-2060.

[4] A. Aldroubi, Non-uniform weighted average sampling and reconstruction in shiftinvariant and wavelet spaces, Appl. Comput. Harmon. Anal. 13 (2002) 151-161.

[5] A. Aldroubi and K. Gröchenig, Non-uniform sampling and reconstruction in shiftinvariant spaces, SIAM Rev. 43 (2001) 585-620.

[6] A. Aldroubi, Q. Sun and W. S. Tang, Convolution, average sampling and a Calderon resolution of the identity for shift-invariant spaces, J. Fourier Anal. Appl. 11(2) (2005) 215-244.

[7] M. S. Birman and M. Z. Solomjak, Spectral Theory of Self-Adjoint Operators in Hilbert Space (Springer, New York, 1987).

[8] O. Christensen, An Introduction to Frames and Riesz Bases (Birkhäuser, Boston, 2003).

[9] O. Christensen and Y. C. Eldar, Oblique dual frames and shift-invariant spaces, Appl. Comput. Harmon. Anal. 17(1) (2004) 48-68.

[10] O. Christensen and Y. C. Eldar, Generalized shift-invariant systems and frames for subspaces, Appl. Comput. Harmon. Anal. 11(3) (2005) 299-313.

[11] I. Djokovic and P. P. Vaidyanathan, Generalized sampling theorems in multiresolution subspaces, IEEE Trans. Signal Process. 45 (1997) 583-599.

[12] H. R. Fernández-Morales, A. G. García and G. Pérez-Villalón, Generalized sampling in $L^{2}\left(\mathbb{R}^{d}\right)$ shift-invariant subspaces with multiple stable generators, in Multiscale Signal Analysis and Modeling, Lecture Notes in Electrical Engineering (Springer, New York, 2012), pp. 51-80.

[13] A. G. García, M. A. Hernández-Medina and G. Pérez-Villalón, Generalized sampling in shift-invariant spaces with multiple stable generators, J. Math. Anal. Appl. 337 (2008) 69-84. 
[14] A. G. García and G. Pérez-Villalón, Dual frames in $L^{2}(0,1)$ connected with generalized sampling in shift-invariant spaces, Appl. Comput. Harmon. Anal. 20(3) (2006) $422-433$.

[15] A. G. García and G. Pérez-Villalón, Generalized irregular sampling in shift-invariant spaces, Int. J. Wavelets Multiresolut. Inf. Process. 5(3) (2007) 369-387.

[16] A. G. García and G. Pérez-Villalón, Multivariate generalized sampling in shiftinvariant spaces and its approximation properties, J. Math. Anal. Appl. 355 (2009) $397-413$.

[17] R. A. Horn and C. R. Johnson, Matrix Analysis (Cambridge University Press, 1999).

[18] S. Kang and K. H. Kwon, Generalized average sampling in shift-invariant spaces, J. Math. Anal. Appl. 377 (2011) 70-78.

[19] A. N. Kolmogorov, Stationary sequences in Hilbert space, Boll. Moskow. Gos. Univ. Mat. 2 (1941) 1-40.

[20] S. Li and H. Ogawa, Pseudo-duals of frames with applications, Appl. Comput. Harmon. Anal. 11 (2001) 289-304.

[21] S. Li and H. Ogawa, Pseudoframes for subspaces with applications, J. Fourier Anal. Appl. 10(4) (2004) 409-431.

[22] T. Michaeli, V. Pohl and Y. C. Eldar, $U$-invariant sampling: Extrapolation and causal interpolation from generalized samples, IEEE Trans. Signal Process. 59(5) (2011) 2085-2100.

[23] A. Papoulis, Generalized sampling expansion, IEEE Trans. Circuits Syst. 24 (1977) 652-654.

[24] V. Pohl and H. Boche, $U$-invariant sampling and reconstruction in atomic spaces with multiple generators, IEEE Trans. Signal Process. 60(7) (2012) 3506-3519.

[25] H. L. Royden, Real Analysis, 3rd edn. (Macmillan Publishing Company, New York, 1988).

[26] M. H. Stone, On one-parameter unitary groups in Hilbert spaces, Ann. Math. 33(3) (1932) 643-648.

[27] W. Sun and X. Zhou, Average sampling in shift-invariant subspaces with symmetric averaging functions, J. Math. Anal. Appl. 287 (2003) 279-295.

[28] M. Unser and A. Aldroubi, A general sampling theory for nonideal acquisition devices, IEEE Trans. Signal Process. 42(11) (1994) 2915-2925.

[29] M. Unser and J. Zerubia, A generalized sampling theory without band-limiting constraints, IEEE Trans. Circuits Syst. II 45(8) (1998) 959-969.

[30] R. Venkataramani and Y. Bresler, Sampling theorems for uniform and periodic nonuniform MIMO sampling of multiband signals, IEEE Trans. Signal Process. 51(12) (2003) 3152-3163.

[31] G. G. Walter, A sampling theorem for wavelet subspaces, IEEE Trans. Inform. Theory 38 (1992) 881-884.

[32] J. Weidmann, Linear Operators in Hilbert Spaces (Springer, New York, 1980).

[33] K. Yosida, Functional Analysis, 6th edn. (Springer, New York, 1995).

[34] J. Zhou, Multidimensional multirate systems characterization, design and applications, thesis, University of Illinois (2005). 